\title{
Zur Mikromorphologie und zum Tonmineralbestand von Böden ungarischer Lößprofile und ihre paläoklimatische Auswertung
}

\author{
Von Arnt Bronger, Kiel
}

Mit 10 Abbildungen und 1 Tabelle

\begin{abstract}
$\mathrm{Z}$ us a mmen f a s sung. Gegenüber den westlichen Nachbargebieten sind Lößprofile in Ungarn durch fossile Böden besonders jungpleistozänen Alters detaillierter untergliedert. Für ihre paläoklimatische Auswertung dienen neben dem Geländebefund einmal mikromorphologische Beobachtungen, die eine genauere Zuordnung der Paläoböden jeweils zu einem Bodentyp als Ausdruck ihrer Genese gestatten. - Untersuchungen zum Tonmineralbestand der Lösse und der jeweils aus ihnen entstandenen Böden ermöglichen nähere Aussagen über das Ausmaß der Ne ubil $\mathrm{dung}$ der einzelnen Minerale in den Tonteilfraktionen und damit unter bestimmten Voraussetzungen Hinweise auf die Intensität und Dauer einer Bodenbildung.

In allen Böden überwiegt die Neubildung des Montmorillonits bei weitem. Sie ist fast ausschließlich auf die Feintonfraktion beschränkt, jedoch sind die hohen Montmorillonitanteile auch in den B-Horizonten höchstens zu einem geringen Teil auf eine Tonverlagerung zurückzuführen - mikromorphologisch nachweisbar dadurch, daß Fließplasma (Braunlehm-Teilplasma) nicht oder nur in Spuren zu beobachten ist. Die Zunahme der einzelnen Tonmineralarten ist in den basalen Waldböden des Pakser Profils nicht wesentlich höher als in den jungpleistozänen Waldsteppenböden (mit Ausnahme des sehr schwachen ", $\mathrm{F}_{4 \mathrm{a}}$ “-Bodens in Mende), in diesen aber mindestens so hoch wie im rezenten Braunerde-Tschernosem, wobei weitere rezente Lößböden in die vergleichende Untersuchung mit einzubeziehen sind.
\end{abstract}

Die jungpleistozänen Waldsteppenböden $\left({ } \mathrm{F}_{4}\right.$ “ bis ${ } \mathrm{F}_{2}{ }$ “) dieses Raumes müssen nach den vorgelegten Untersuchungsergebnissen als Zeugen nicht eines interstadialen, sondern eines warmzeitlichen Klimas etwa vergleichbar dem des heutigen angesehen werden.

$\mathrm{Summary}$. In Hungary loess profiles are subdivised by paleosols especially of upper pleistocene age in much greater detail than in adjacent western countries. Paleoklimatic interpretations are based on field studies as well as on micromorphological observations, which permit an exact classification of paleosols regarding soil type and genesis. Investigations of the clay mineral contents of loess and of the various soils developing from loess show the amount of formation of clay minerals in the different particle size fractions and thus supply under certain conditions information about the intensity and duration of soil formation.

In all soils examined here the formation of montmorillonites is by far the largest. It is almost entirely limited to the fine clay fraction $(<0,2 \mu)$, however the large amount of montmorillonites in the B-horizons can be explained only to a small extent by clay illuviation. Illuviation, which is normally indicated micromorphologically by the existence of "Fließplasma (Braunlehm-Teilplasma)" was not found in these samples. The increase of clay mineral contents in the basic forest soils of the Paks profile is not much greater than in the upper pleistocene forest-steppe-soils (except in the weak " $\mathrm{F}_{4 \mathrm{a}}$ "-soil in Mende). In these the increase is at least as high as in the recent Braunerde-Tschernosem. However, other recent loess soils will have to be examined in comparison.

The upper pleistocene forest-steppe-soils $\left({ } \mathrm{F}_{4}\right.$ “ to ${ } \mathrm{F}_{2}$ “) of this area must be considered according to these investigations not as proof of an interstadial but of a warm climate, comparable to our present one.

\section{Problemstellung und Zielsetzung der Untersuchungen}

Anläßlich der Arbeitstagung der Subkommission für Lößstratigraphie der INQUA 1965 konnten Lößprofile Ungarns studiert und in einem kleinen Fachkreis diskutiert werden. Die hier sehr detaillierte Abfolge von Paläoböden, besonders innerhalb der oberpleistozänen Lösse, entsprach so wenig derjenigen anderer Lößprofile Mitteleuropas, daß unter den Kommissionsteilnehmern beträchtliche Meinungsunterschiede über die bodentypologische Ansprache, die klimatische Bewertung und zeitliche Einordnung der fossilen Böden bestanden. Insbesondere die Frage, welcher fossile Boden das letzte Interglazial (Riß/ 
Würm) markiert, war Gegenstand von ausgedehnten Diskussionen (s. Diskussionsmaterial etc. 1965).

Für die Rekonstruktion einer Klimageschichte des Pleistozäns auf paläopedologischer Grundlage ist einmal eine möglichst genaue Zuordnung der fossilen Böden jeweils zu einem Bodentyp notwendig. Diese Zuordnung orientiert sich an den rezenten Böden. Jedoch sind manche Kriterien für die typologische Ansprache von rezenten Böden für fossile Böden nur noch mit Vorbehalten oder gar nicht mehr anwendbar. Selbst wenn Paläoböden nicht nach ihrer Bildung umgelagert wurden, liegen sie oft nur als mehr oder weniger stark gekappte Bodenreste vor. Jedenfalls ist bei Waldböden der im allgemeinen geringmächtige A-Horizont, meistens auch noch ein Teil des Unterbodens der späteren Abtragung zum Opfer gefallen. Der mit zunehmendem Alter fortschreitende Abbau der organischen Substanz (z. B. BRONGER 1966a, 1969b) erschwert auch bei weitestgehender Erhaltung eine makroskopische Horizontdifferenzierung.

Dazu sind manche bodenchemische Untersuchungsergebnisse, die mit Horizonteinheiten bzw. Humuswerten in einer Korrelation stehen, beispielsweise die von pedogenen Eisenoxiden (z. B. Schlichting und Blume 1962, SCHwertmann 1964) nur noch beschränkt ausdeutbar. Analysen u. a. des „freien“ (dithionitlöslichen) Eisengehaltes — das „als brauchbarer Maßstab für die Charakterisierung der lösungschemischen Verwitterung" gilt (Rohdenburg und Meyer 1966, 90) - ergaben zwar bei den heutigen Lößböden des Karpatenbeckens etwa die bekannten Korrelationen der $\mathrm{Fe}_{\mathrm{d}}$-Werte mit den Horizonten der verschiedenen Bodentypen, vor allem mit der jeweiligen Tonneubildungsrate. Dagegen wurden in den weiter unten beschriebenen Lößböden der Profile von Paks und Mende sowie in Paläoböden jugoslawischer Lößprofile im allgemeinen keine entsprechenden signifikanten Werte gefunden, was vielleicht auf bisher wenig beachtete Alterungsprozesse in den Paläoböden zurückgeführt werden könnte. In ähnlicher Richtung wiesen die sehr geringen Aktivitätsgrade der Eisenoxide $\left(\mathrm{Fe}_{\mathrm{o}} / \mathrm{Fe}_{\mathrm{d}}\right.$-Werte nach SchWERTMANN 1964) von stets $<0,1$ : hier dürfte als Alterungsprozeß die Kristallisation eines Teiles der aktiven amorphen Eisenoxide in Betracht kommen. An anderer Stelle wird darüber näher berichtet werden.

Erschwert werden Ausdeutungen von bodenchemischen Analysen aber vor allem durch die fast stets erfolgende sekundäre Aufkalkung der fossilen Böden infolge Überlagerung von Löß. So geben die $\mathrm{pH}$-Werte von Paläoböden - z. B. im Profil von Paks stets über 7 (Kriván 1955b) oder in den Waldböden des Bugginger Profils (Südbaden) selbst in 0,1n KCl über 6 (BRONGER 1969b) - nur diesen sekundär veränderten, verfälschten $\mathrm{Zu}$ stand wieder, entsprechend verhält es sich z. B. mit Sättigungsgraden (V-Werten). Der sekundäre Kalkgehalt erschwert insbesondere die Unterteilung des Tschernosems in Subtypen.

Schließlich ändert sich die Struktur eines begrabenen Bodens in Richtung auf eine Gefügeverdichtung, die mit zunehmender Mächtigkeit der Überlagerung (in unserem Fall bis über $40 \mathrm{~m}$ ) und wohl auch mit fortschreitendem Alter zunimmt. Ein weiterer Faktor dürfte der in unseren Beispielen im allgemeinen relativ hohe Tongehalt der Lösse, erst recht der aus ihnen entstandenen Böden sein. So kann aus einem ehemaligen Krümelgefüge durch Reduzierung der Hohlräume ein mehr oder weniger stark verdichtetes Kohärentgefüge werden, was im Einzelfall weiter unten mikromorphologisch näher beschrieben wird. - Insgesamt kommt neben der makroskopischen Profilansprache der mikromorphologischen Untersuchung für die Zuordnung des fossilen Bodens zu einem Bodentyp besondere Bedeutung zu. Sie erlaubt zudem das Erkennen von polygenetischen Entwicklungen der Paläoböden (Kubiena 1956, 1964, Smolíkoví 1967, 1968, Bronger $1966,1969 b)$.

Für das oben angedeutete Problem der Rekonstruktion einer Klimageschichte des Pleistozäns auf paläopedologischer Grundlage und speziell für die Frage, welcher Boden das 
letzte Interglazial repräsentiert, ist es notwendig, gesicherte Hinweise auf die Intensität und Dauer einer Bodenbildung zu erhalten. Dafür ist ein großer Teil der üblichen chemischen Untersuchungsmethoden, wie dargelegt, weniger brauchbar. Bei bisherigen Untersuchungen über Verwitterung und Bodenbildung an westmitteleuropäischen Lößprofilen (Schroeder 1954, 1955, Bronger 1966, Bronger, Graf v. Reichenbach \& Schroeder 1966, Bronger 1969a, b) hat sich vor allem die Analyse des Tonmineralbestandes als eine wertvolle Hilfe erwiesen. Insbesondere bei einer weiteren Aufteilung der Tonfraktion sind - unter gleichzeitiger Berücksichtigung der Korngrößenverteilung der Lösse und der aus ihnen entstandenen Böden - nähere Aussagen über das Ausmaß der N e u bil d ung der einzelnen Mineralarten möglich. Die Gleichartigkeit des Ausgangsmaterials sowie der mikromorphologische Befund, daß in zwei Paläoböden höchstens eine äußerst geringe, in allen anderen Böden überhaupt keine Tonverlagerung stattfand, erlaubt dazu die Aufstellung von angenäherten Verwitterungs(teil)bilanzen der einzelnen Paläoböden und ihren Vergleich untereinander.

In den Jahren 1966-1968 wurden neben anderen Geländearbeiten mehrere Lößaufschlüsse in Ungarn und Jugoslawien besonders entlang der Donau untersucht, um auf bodengeographischer Grundlage einmal eine quartäre Klimageschichte des Karpatenbeckens erstellen zu können. Hier soll über Untersuchungsergebnisse zur Mikromorphologie und zum Tonmineralbestand von Böden der Lößprofile von Paks und Mende sowie des basalen Bodens des Profils von Nogradveröce berichtet werden. Zum Vergleich wurde ein rezenter Braunerde-Tschernosem aus Löß als charakteristischer Bodentyp des Hügellandes von Gödöllö-Monor herangezogen.

Bei den Geländearbeiten unterstützten mich der Direktor des Geographischen Instituts der Ungarischen Akademie der Wissenschaften, Prof. Dr. M. Pécsi mit seinen Mitarbeitern, insbesondere Dr. Gy. Hahn, Dr. S. Marosi und Dr. J. Szilárd, wofür ihnen herzlicher Dank gesagt sei. Die Deutsche Forschungsgemeinschaft half bei zwei der Reisen mit Reisebeihilfen und finanzierte die Herstellung der Dünnschliffe.

Die Herstellung der sehr zahlreichen Texturpräparate und Röntgenaufnahmen besorgten Frau Lenthe und Herr Hinze; Frau Lenthe fertigte darüber hinaus die Reinzeichnungen der Abbildungen 1, 2 und 10 an. Ihnen sei auch an dieser Stelle herzlich gedankt.

\section{Untersuchungsergebnisse}

\subsection{Die Lößprofile von Paks, Mende und Nogradveröce} Ein holozäner Boden als Vergleich

An dieser Stelle mag es genügen, die zahlreiche Literatur über das Lößprofil von Paks nur in aller Kürze auf die hier interessierenden Fragen hin anzuführen. Schon 1934 benutzte Bulla diesen Aufschluß zur Untergliederung einzelner Lößakkumulationsphasen, die er mit der Bildung von Flußterrassen gleichzusetzen versuchte. Später (1937) erkannte er wie schon ScHERF (1936) in den fossilen Böden die "B-Horizonte alter Waldböden" von Interglazial- bzw. Interstadialcharakter mit regionaler (nicht lokaler) Bedeutung. Seine stratigraphische Gliederung ging - wie die von Scherf (1936) - von der Dreiteilung der Würmeiszeit nach Soergel aus.

In der Folgezeit stand die Untergliederung des Pleistozäns im allgemeinen, damit die Zuordnung einzelner Schichtenkomplexe des Pakser Profils zu Abschnitten des Pleistozäns im besonderen stark unter dem Einfluß der astronomischen Theorie der Klimaschwankungen von Milankovič und Bacsák (1955), etwa die Gliederung von Adam, Marosi und Szillárd (1954). Honváth (1954) bearbeitete die Gastropodenfauna dieses Profils in sehr zahlreichen (im Abstand von $20 \mathrm{~cm}$ ), aber kleinen Proben (jeweils nur einige $100 \mathrm{~g}$ ), so daß meistens nur wenige Individuen angeführt wurden. Obwohl er in den oberen $35 \mathrm{~m}$ des Profils keine thermophilen Arten fand, wurde der - nicht ohne weiteres ersichtliche - periodische Wechsel der Fauna mit den Klimaschwankungen nach der MiLANkovič-BACsék'schen Theorie in Übereinstimmung gebracht. Ähnlich betrachtete Kriván (1955a) diese Theorie „als eine exakte Basis der klimatischen Quartärgliederung" (S. 379), wobei er die fossilen Böden in Paks offensichtlich als feucht-kaltzeitliche Bildungen ansah (S. 379). In seiner Monographie, der bisher detailliertesten Untersuchung dieses Profils (1955b) wurden andererseits zahlreiche Proben in engem Abstand nach Korngröße1), pH-Wert

1) Vgl. Anmerkung 8, S. 140. 
und Kalkgehalt analysiert. Während alle genannten Bearbeiter vor ihm das Pakser Profil für vollständig hielten, nahm KRIVÁN an, daß die ältesten pleistozänen Schichtserien bereits das Günz/ Mindel-Interglazial repräsentieren.

Eine nähere Beschreibung auch der fossilen Böden mit chemischen und mikromorphologischen Angaben sowie einigen DTA-Kurven der Fraktion $<2 \mu$ finden sich dann bei STEFANovits und RozSAvÖGYI (1962), wobei die Paläoböden jedoch nicht typisiert werden - in der Profildarstellung sind nur "Bodenhorizonte“ (mit einheitlicher Signatur) und „schwache Horizonte“ unterschieden. Erst PÉCSI (1965a, 1966) versuchte - vom makroskopischen Befund ausgehend - eine typologische Zuordnung der fossilen Böden des Pakser Profils. - Von ihm wurde ebenfalls zuerst das Lößprofil von Mende, $35 \mathrm{~km}$ südöstlich Budapest im Hügelland von Gödöllö-Monor (neben zahlreichen weiteren Lößprofilen im Karpatenbecken) bearbeitet (1965c, 1966). Vom Profil von Mende wurden von Stefanovits (1965) Korngrößen- und chemische Analysen durchgeführt, worauf wir später noch zurückkommen.

Vom Lößprofil von Paks ist die Abfolge von Lössen und fossilen Böden nur noch im südlichen Teil des Aufschlusses sichtbar. Dabei waren einzelne Abschnitte in den Jahren 1966, 1967 und 1968, in denen das Profil immer wieder aufgesucht wurde, unterschiedlich gut $\mathrm{zu}$ bearbeiten, beispielsweise der unterste Boden $\left.\left({ } \mathrm{F}_{8} “\right)^{2}\right)$ nur im Sommer 1967, der zweitunterste $\left.\left({ } \mathrm{F}_{7}{ }^{\prime}\right)^{2}\right)$ in größerer horizontaler Erstreckung im Sommer 1966. - Vom Lößprofil der Ziegelei in Mende, 1965 anläßlich der Tagung der Subkommission noch gut aufgeschlossen, waren in den nächsten Jahren nur noch Teile aus dem oberen Abschnitt zu sehen. Jedoch waren besonders 1966 in einem wenige $100 \mathrm{~m}$ entfernt gelegenen Lößhohlweg die fossilen Böden gut aufgeschlossen, insbesondere bei dem hier als „ $\mathrm{F}_{3}$ “ bezeichneten Boden ("Mende D-Boden“ nach PÉCSI 1965c) unter dem A-Horizont ein geringmächtiger (B)-Horizont, der sich farblich aber deutlich absetzt. Die Profilzeichnung (Abb. 4) des Lößproenls von Mende gibt ein kombiniertes Bild (schematisiert) der beiden Profile wieder. Der unterste Teil des Aufschlusses bei der Ziegelei mit dem basalen Bodenkomplex („Mende H- und I-Boden“ nach PÉCSI 1965c) konnte erst im Herbst 1968 wieder aufgegraben und deshalb noch nicht tonmineralogisch untersucht werden. Deshalb wurde der basale „Bodenkomplex" des in seiner hangenden Löß-Boden-Abfolge nicht so vollständigen Profils von Nogradveröce, $30 \mathrm{~km}$ nördlich Budapest, der dem des Profils von Mende stratigraphisch entsprechen dürfte, in die vergleichende Untersuchung mit einbezogen. - Da der rezente Boden in der Umgebung von Mende infolge beträchtlichen Mikroreliefs und Kulturmaßnahmen stark der Abtragung unterliegt, wurde im wenige km benachbarten Tapiosüly ein Braunerde-Tschernosem vergleichend untersucht.

Von diesem rezenten und den fossilen Böden sowie den unterlagernden Lössen der genannten Lößprofile wurden größere Proben für physikalische, chemische und mineralogische Untersuchungen, außerdem aus den verschiedenen Horizonten Rahmenproben der Lösse und Böden in natürlicher Lagerung zur Herstellung von Dünnschliffen für die mikromorphologische Untersuchung entnommen.

Die genannten Lößaufschlüsse sind in den Abbildungen 1 und 4 schematisch dargestellt. Im Hinblick auf die Zielsetzung dieser Arbeit mag es daher genügen, unter besonderer Berücksichtigung der mikromorphologischen Untersuchungsergebnisse kurz auf die Genese der Paläoböden näher einzugehen. Die bodentypologische Einordnung richtet sich einerseits soweit wie möglich nach der Systematik von Mückenhausen (1962) ${ }^{3}$ ), obwohl in Ungarn genetische Klassifikationen der Wald- und Steppenböden vorliegen (STEFANOvits

2) Die Indizes sollen hier die stratigraphische Position kennzeichnen, worauf im letzten Abschnitt noch eingegangen wird. Sie stimmen mit denen PÉCsIs (1966) oft nicht überein.

3) Dabei sei besonders auf die Bemerkung von MüCKEnHAuSEN $(1962,74)$ hingewiesen, wonach es noch nicht möglich ist (was leider auch heute noch gilt), die Klasse der Braunerden - und das trifft ähnlich auch für die Steppenböden $\mathrm{zu}$ - allgemeingültig voneinander abzugrenzen, insbesondere nicht, weil die bisher entwickelten Konzeptionen unzureichend aufeinander abgestimmt sind. Das wird bei Diskussionen an Aufschlüssen, z. B. anläßlich von Tagungen, immer besonders deutlich. 
1959b, Szücs 1959, Stefanovits und Szücs 1961) ${ }^{4}$ ). Andererseits mußte die Abgrenzung einiger Typen und Subtypen nach der mikromorphologischen Definition von KubienA (1956, 1964) erfolgen, vor allem, weil bei stärker gekappten Waldböden nur eine mikromorphologische Unterscheidung von Braunerden und Parabraunerden eindeutig ist (s. auch BONGER 1969c) und auch, weil z. B. braunlehmartige Böden dort (1964) näher gekennzeichnet sind.

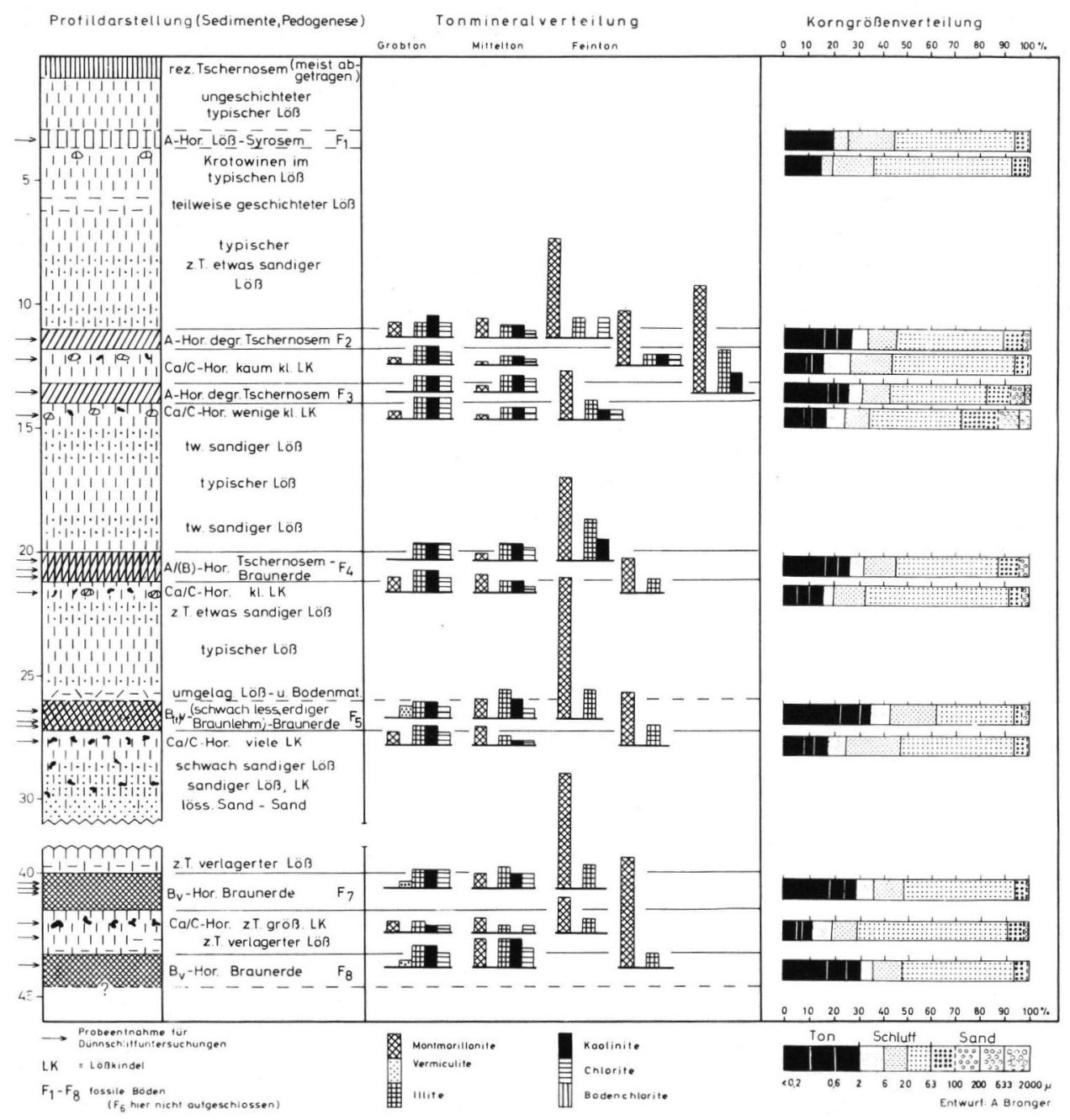

Abb. 1. Lößprofil von Paks.

\section{Das Lößprofil von Paks}

Die beiden untersten Paläoböden ( $\mathrm{F}_{7}$ “ und „ $\mathrm{F}_{8}$ “) sind sich sowohl nach Farbe und Gefüge wie nach ihrer Mikromorphologie außerordentlich ähnlich. Sie sind von auffallend

4) Diese Klassifikationen berücksichtigen insbesondere den jetzt noch oder vor den Meliorationsperioden vorhandenen Grundwassereinfluß, der wegen der Versalzungsgefahr natürlich ein äußerst wichtiger Faktor für die rezenten Böden in den tiefer gelegenen Teilen der Kleinen und Großen Ungarischen Tiefebene darstellt. 
rötlichbrauner Farbe (5 YR 5/4) ${ }^{5}$ ), die sich deutlich von der rezenter Waldböden aus Löß unterscheidet. Die mächtigen, noch vorhandenen Unterböden haben ein dichtes Kohärentgefüge und sind anstehend sehr stark verhärtet. Die Böden sind sekundär aufgekalkt, kenntlich an oft zentimetergroßen Bieloglaska besonders im ${ }_{\mathrm{F}_{7}}$ “.

Mikromorphologisch zeigt sich einmal die gute Durchflockung der rötlich braunen Feinsubstanz im dichten Braunerdegefüge. Häufig umgibt sie in weiten Bögen heller gefärbte Teile des Grundgefüges, in welchem der Skelettanteil höher ist (Abb. 3). Es handelt sich aber sicher nicht um ehemalige Leitbahn(reste), denn abgesehen davon, daß diese feinsubstanzangereicherten Partien keine Richtung bevorzugen, fehlt - auch stark granuliertes und vererdetes - Braunlehmteilplasma. Möglicherweise sind es Spuren von Organismen, jedoch ist das Gefüge sehr hohlraumarm, Intergranularräume sind kaum vorhanden, so daß auch eine Aggregatbildung nur in Form von Mikroaggregaten an einigen Stellen zu erkennen ist. Andererseits fehlen aber auch Sprungrisse. Die Hohlraumarmut dürfte mindestens teilweise durch die hohe Auflast (über $40 \mathrm{~m}$ ) verbunden mit einem relativ hohen Alter dieser Böden zu erklären sein.

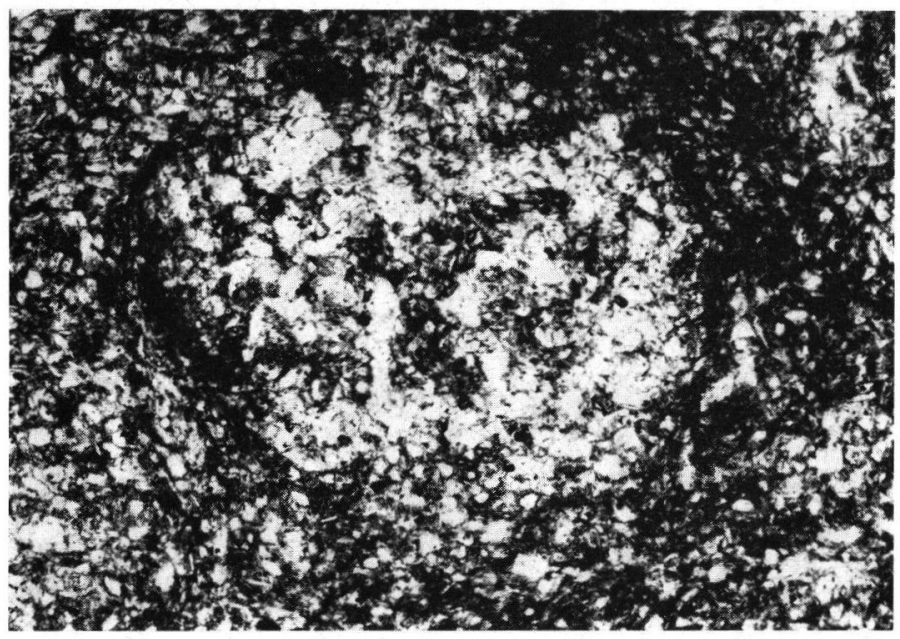

Abb. 2. Zweitunterste fossile Braunerde („F $\mathrm{F}_{8}$ “) im Lößprofil von Paks. - Hellere, skelettreichere Teile des gut durchflockten, aber hohlraumarmen Braunerdegefüges, umgeben von dunklerer, tonreicherer Feinsubstanz, vermutlich Spuren von Organismen. (Hellfeld, Vergrößerung 32-fach.)

Mit der beschriebenen, von heutigen Löß-Waldböden abweichenden Merkmalen sind sie typologisch als $B_{v}$-Horizonte von gut durchflockten Braunerden einzuordnen.

Der Löß zwischen den beiden Böden besitzt eine heterogene Mineralzusammensetzung (insbesondere Quarze, Feldspäte, Muskovite, Biotite, letztere meist mit starkem Pleochroismus); er ist gleichmäßig mit Kalzitkristallen durchsetzt. Die sekundäre Aufkalkung vom hangenden Boden reicht bis weit über $1 \mathrm{~m}$ hinab und ist kenntlich an den unzähligen Kalzitmikrolithen in den wenigen Hohlräumen. Einige große (mehrere $100 \mu \phi$ ), an früherer Stelle (BRONGER 1966a) näher beschriebene, aus mehreren Kalzitkristallen zusammengewasene Kalzitkörner, Ausscheidungen wahrscheinlich von Lumbriciden (Abb. 8) zeigen an, daß der Löß nicht unbelebt war. - Auf die im ganzen ähnliche Mikromorphologie der $\mathrm{Ca} / \mathrm{C}$-Horizonte der hangenden Paläoböden sei der notwendigen Kürze wegen nur summarisch hingewiesen.

5) Alle Munsell-Werte beziehen sich auf trockene Proben. 
Von dem als „ $\mathrm{F}_{5}$ “ bezeichneten Boden ist nur noch der untere Teil $(60 \mathrm{~cm})$ des Unterbodens ungestört erhalten; der obere Teil (ebenfalls $60 \mathrm{~cm}$ ) ist zunehmend mit hangendem Löß vermischt. Der untere Abschnitt ist (rötlich) braun gefärbt (7,5 YR 5/5) und enthält im untersten Teil zentimetergroße weiße Kalkausscheidungen, sowohl in rundlicher Form (Bieloglaska) als auch vertikal langgestreckt, sicherlich eine Folge sekundärer Aufkalkung. Das Gefüge ist undeutlich subpolyedrisch und etwas hohlraumreicher. - Der Ca/C-Horizont ist kräftig entwickelt, die lößkindelfreien Teile haben immer noch über $30 \% \mathrm{CaCO}_{3}$.

Mikromorphologisch zeigt sich ein relativ hohlraumarmes Braunerdegefüge, was hier deutlicher als bei den beiden basalen Böden zu einem rötlichbraun gefärbten dichteren, erdigen Braunlehmgefüge mit einigen Sprungrissen überleitet. Nur an ganz wenigen Stellen sind sehr kleine Teile von Braunlehmteilplasmarestchen, meist auch granuliert, mit abnehmender Doppelbrechung sichtbar. Sie sind nachfolgend oft überdeckt von kleinen aber konzentrierten Fe-Mn-Oxiden (i.w.S.). Erwähnt seien schließlich Aggregate in Teilen der Grundmasse, deren größere meist scharfkantig umgrenzt sind. - Typologisch ist dieser Bodenrest als $\mathrm{B}_{(\mathrm{t})_{\mathrm{v}}}$ oder $\mathrm{B}_{\mathrm{v}}$-Horizont einer ( $\mathrm{schwach}$ lessivierten, erdigen B ra un lehm) - B ra un e rde anszusprechen.

Obwohl der durch ca. $5 \mathrm{~m}$ Löß getrennte, hangende Boden $\left({ } \mathrm{F}_{4}\right.$ “) annähernd vollständig erhalten blieb, ist seine typologische Einordnung nicht leicht. Seine durchgehend (schwächer rötlich-)braune Farbe (7,5 YR 4/4) und Krotowinen im liegenden Löß haben PÉCSI vielleicht veranlaßt, ihn einmal als „rosabraunfarbigen Steppenboden“ bzw. „kastanienfarbigen Boden“ (1966), an anderer Stelle als einen „Kastanosem“ (1965b, S. 77) bzw. „chestnut-brown soil“ (1967) zu bezeichnen. Jedoch sprechen schon seine Mächtigkeit (ca. $1,20 \mathrm{~m}$ ) und der $\mathrm{Ca} / \mathrm{C}$-Horizont mit weitständig fautsgroßen Lößkindeln nicht für diese Deutung, ebenso die Zunahme der Tonfraktion von gut 10\% (Tab. 1). Kastanoseme bzw. kastanienfarbene Tschernoseme Südrumäniens haben nur einen wenige Prozent $(2-4 \%)$ höheren Tongehalt als der unterlagernde Löß (Exkursionsführer, S. 179-187). Südrussische Löß-Kastanoseme zeigen mikromorphologisch ein sehr porenreiches, gut aggregiertes Gefüge (Short Guide etc., S. 69). Dagegen erweist sich das hier anstehend sehr dichte Kohärentgefüge mikromorphologisch als hohlraumarmes, im Vergleich zu den basalen Böden skelettreiches, durchflocktes Braunerdegefüge, mit nur in kleinen Teilen undeutlicher Aggregatbildung. Die wesentlich geringere Verwitterungsintensität zeigt sich u. a. in den vielen Biotiten, die meist noch Pleochroismus aufweisen. Da insgesamt die Merkmale einer (schwächer verwitterten) Braunerde gegenüber makroskopisch sichtbaren eines Steppenbodens überwiegen, außerdem das Stratum nicht in einzelne Horizonte untergliederbar ist, soll dieser Boden als A/(B)-Horizont einer Ts chernose m-B raunerde angesprochen werden.

Die beiden hangenden Böden ( $\mathrm{F}_{3}$ “ bzw. „ $\mathrm{F}_{2}$ “) sind wieder makro- wie mikromorphologische recht ähnlich. Die dunkelbraunen (7,5 YR 4/4) A-Horizonte, die noch nicht so stark an Humus verarmt sind wie die liegende Tschernosem-Braunerde, haben ebenfalls ein recht dichtes Korärentgefüge, was in Teilen aber bereits krümelig ist. Der untere der beiden Böden zeigt häufig an Kluftlächen schwarze Fe-Mn-Ausscheidungen, mikromorphologisch sind diese jedoch nur kleinflächig und vereinzelt sichtbar. - Mikroaggregate sind wegen der Hohlraumarmut nur in Teilen vorhanden (Abb. 3). Diese ist z. T. sicherlich ebenfalls auf eine noch beträchtliche Überlagerung (11 bzw. $13 \mathrm{~m})$, verbunden mit einem höheren Tongehalt zurückzuführen. Der obere der beiden Böden ist kalkfrei, mit einem deutlichen sehr blaßbraunen (10 YR 7/3) Ca-Horizont, in dem sich einige Krotowinen stark abheben, aber nur vereinzelten, sehr kleinen Lößkindeln, wie der untere Boden, dessen geringer Kalkgehalt (ca. 3\%) sicher sekundär ist. Zahlreicher sind die Biotite, meist noch mit Pleochroismus. Beide Böden (der untere stärker geköpft) sind als d e g r a dierte Tschernoseme mit geringer Pseudovergleyung anzusprechen. - Bei allen 
drei Böden „ $\mathrm{F}_{4}$ “ bis „ $\mathrm{F}_{2}$ “ dürfte es sich um zonale Böden der Waldsteppe handeln. Tonverlagerung in Form des Braunlehm-Teilplasmas ist in keinem Fall zu beobachten.

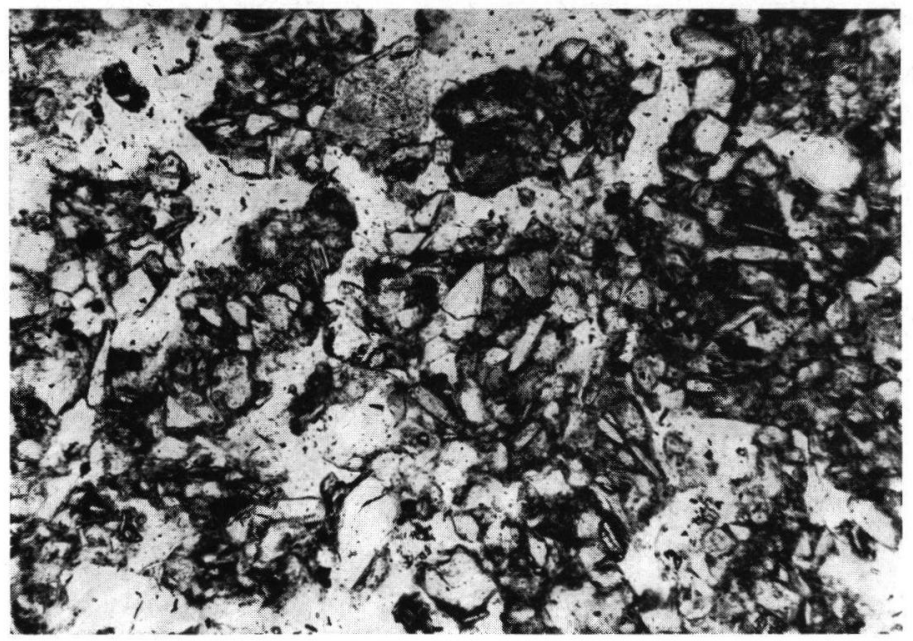

Abb. 3. Unterer fossiler degradierter Tschernosem („F $\mathrm{F}_{3}$ “) im Lößprofil von Paks. - Schorfigkrümelige Mikroaggregate in den wenigen hohlraumreicheren Teilen des sonst porenarmen Gefüges. (Hellfeld, Vergrößerung 80 -fach.)

Der oberste blaßbraune (10 YR 6/3), pedogen wenig überprägte L ö ß-S y r o s em $\left({ }^{\prime} \mathrm{F}_{1}\right.$ “) hat ein leicht zerbröckelbares Kohärentgefüge. Mikromorphologisch zeigt es sich in Teilen als lockeres Schwammgefüge mit meist kleinen Aggregaten, andere Teile sind mehr verdichtet. Die Kalzite und Schneckenschalenreste sind oft schon stärker angelöst. Krotowinen unter diesem bis $70 \mathrm{~cm}$ mächtigen Löß-Rohboden heben sich deutlich vom Löß ab. Dieser Boden ist in stratigraphisch aequivalenter Position in mehreren Profilen im südlichen Teil des Karpatenbeckens sichtbar.

\section{Das Lößprofil von Mende}

Aus den erwähnten Gründen konnte der „basale Bodenkomplex“ („Mende H und I“ nach PÉCSI 1965c) tonmineralogisch noch nicht untersucht werden; er fehlt daher in Abb. 2. Einige makro- und mikromorphologische Angaben sollen hier schon gemacht werden.

Der obere Teil $(100 \mathrm{~cm}$, die obersten $10 \mathrm{~cm}$ Übergang vom hangenden Löß) stellt den A-Horizont eines (degradierten) Ts cher nosems dar, dessen Gefüge relativ locker, undeutlich subpolyedisch bis krümelig (im bergfeuchten Zustand) ist. Die Farbe ist - wohl infolge Humusentfernung - braun (7,5 YR 5/4-4/4) und wird nach unten etwas heller (7,5 YR 5/4), so daß sich eine Krotowine mit Material aus dem oberen A-Horizont deutlicher abhebt. Mikromorphologisch ist die Aggregatbildung trotz Komprimierung in den meisten Teilen gut sichtbar (Abb. 5), Hohlräume sind nicht so selten. Einige von ihnen sind sekundär mit Kalzitmikrolithen wandständig oder ganz ausgekleidet. Im untersten Teil mehren sich bis cm-große Kalkausscheidungen (Bieloglaska). - Obwohl der Übergang zum liegenden Boden ( $80 \mathrm{~cm}$ mächtig) kontinuierlich ist, besteht kein $Z_{\text {weifel, daß }}$ es sich hier um einen echten Pedokomplex handelt, denn der obere und mittlere Teil dieses Horizontes ist stark mit sekundärem Kalk inkrustiert, die weißen, schneidbaren Ausscheidungen erreichen $10 \mathrm{~cm}$ Länge. Infolgedessen muß es sich beim hangenden Tschernosem mindestens zum Teil um eine Neubildung aus frisch angewehtem kalkhaltigen Löß han- 


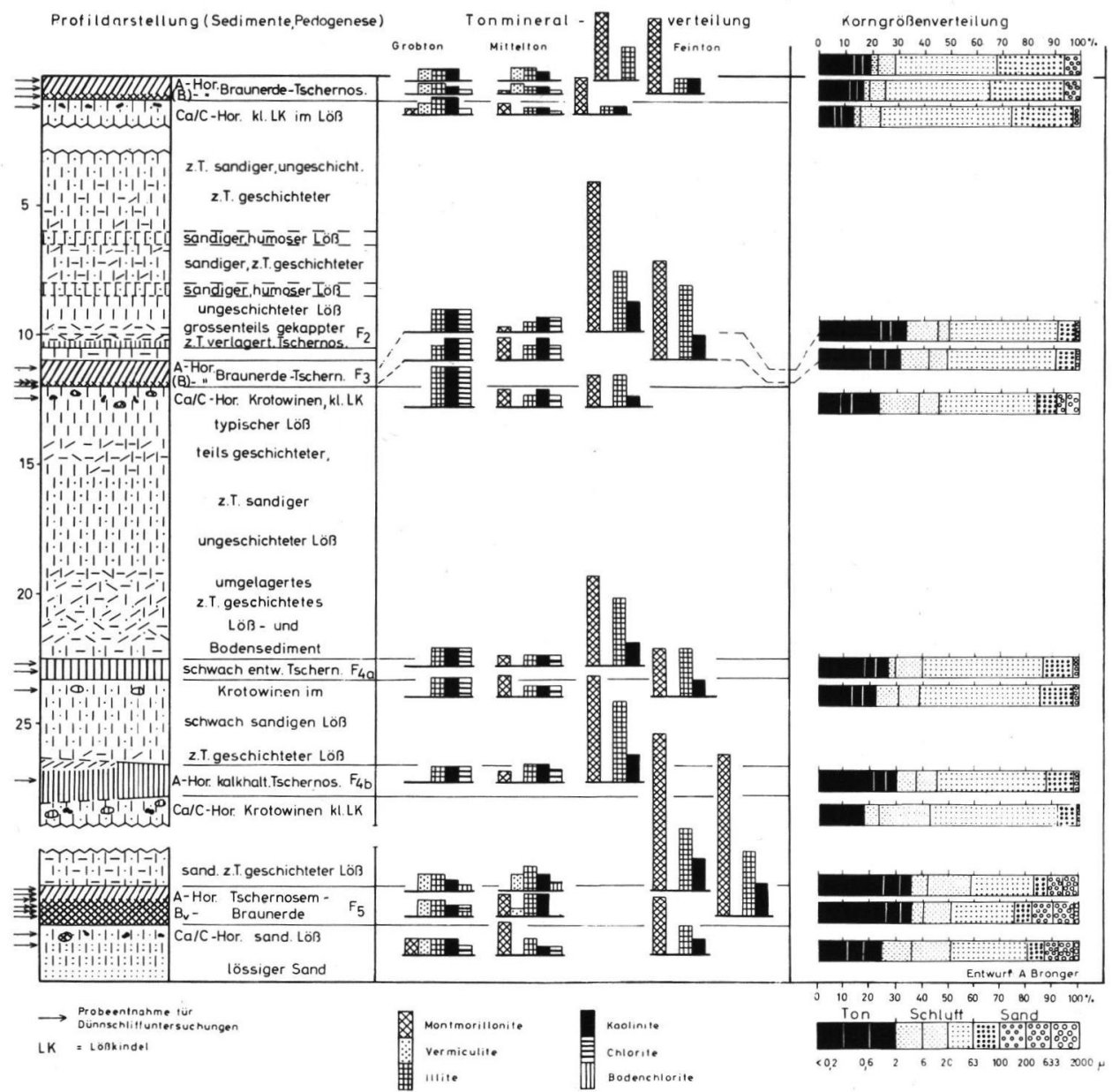

Abb. 4. Lößprofile von Tapiosüly - Mende - Nogradveröce.

deln. - Dieser unterste Boden hat ein mehr scharfkantig-feinpolyedrisches Gefüge. Der oberste Teil enthält mikromorphologisch viele große, verhältnismäßig scharf umgrenzte Aggregate neben kleineren rundlichen Formen. Die zahlreichen Leitbahnen sind völlig frei von Schlämmstoffen (Abb. 6), so daß es sich hier um den $\mathrm{A}(\mathrm{l})$-Horizont bzw. Reste von ihm handelt. Der liegende, farblich kaum abgesetzte, kräftig braune (7,5 YR 5/6, im untersten Teil 7,5 YR 5/6 bis 6/6) Teil enthält in geringen Mengen vom typischen Braunerdegrundgefüge deutlich abgesetztes Braunlehm-Teilplasma, bei dem alle Übergänge zwischen beweglichem, wanderständig geschichteten, stark doppelbrechenden und granuliertem bis vererdetem im Grundgefüge weitgehend vereinnahmten Plasma mit stark abgenommener Doppelbrechung zu beobachten sind. Insgesamt ist dieser unterste Boden als $\mathrm{sch}$ w a ch d rchschlämmte Parabraunerde anzusprechen.

Der nächsthöhere Boden $\left({ } \mathrm{F}_{4 \mathrm{~b}}{ }^{“}\right)^{6}$ ) hier, „Mende-G“ nach PÉCSI (1965), in seinen oberen Abschnitten kolluvial verdickt, hat ein subpolyedrisches, in Teilen undeutlich krümeliges Gefüge von dunkelbrauner Farbe (10 YR 5/3). Mikromorphologisch ist es in

6) Auch der "F $F_{4}$ “-Boden des Pakser Profils war nach PÉCSI (1966, Tafel 32a) früher in Teilen des Aufschlusses durch einen „Gehängelöß“ zweigeteilt. 


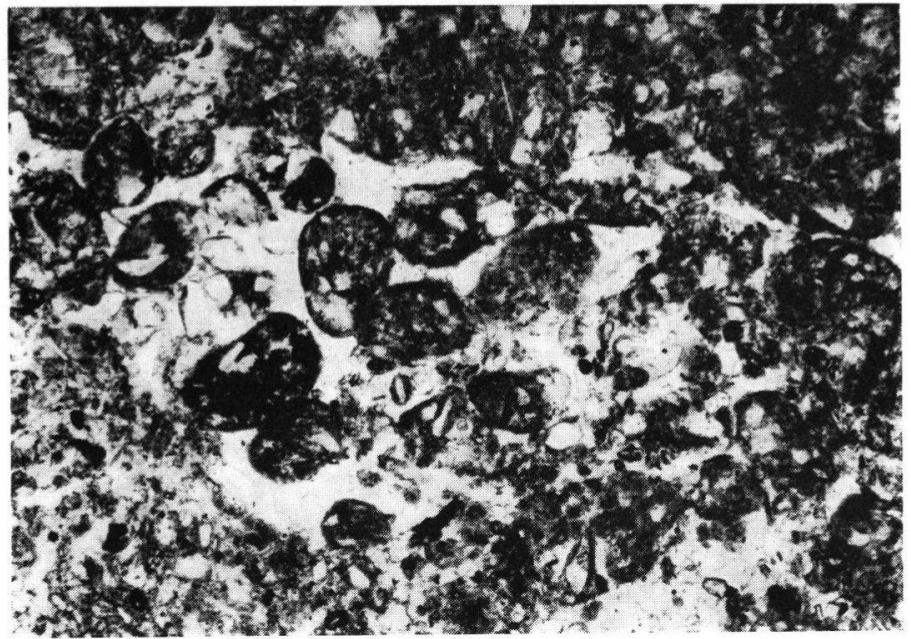

Abb. 5. Oberer Tschernosem des „basalen Bodenkomplexes“ im Lößprofil von Mende. - Zahlreiche gut gerundete Mikroaggregate zeugen von einer einst hohen biologischen Aktivität. (Hellfeld, Vergrößerung 50-fach.)

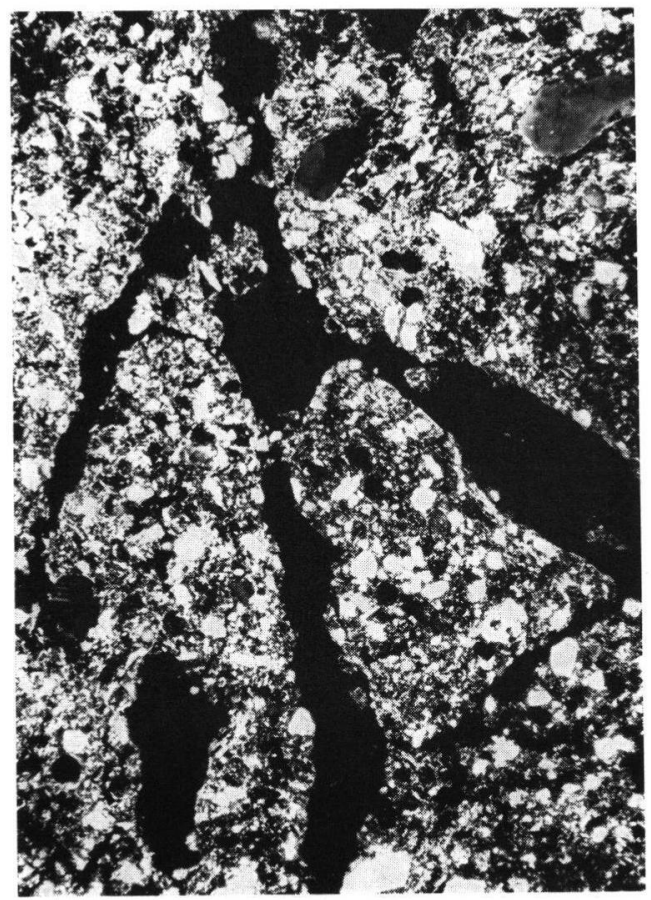

Abb.6. Untere Parabraunerde des „basalen Bodenkomplexes“ im Lößprofil von Mende. Langgestreckte Hohlräume (Leitbahnen) völlig frei von Schlämmstoffen, kennzeichnend für den Eluvial $\left(A_{1}\right)$-horizont einer Parabranerde. ( $+N$, Vergrößerung 32-fach.) 
Teilen stärker verdichtet, in den hohlraumreicheren Teilen sind viele kleine und größere Aggregate recht gut sichtbar. Hier erscheint die Wahrscheinlichkeit besonders groß\}, daß ein ehemaliges Krümelgefüge durch Auflast von 6-8 $\mathrm{m}$ im Hohlwegprofil über längere Zeit, verbunden mit relativ hohem Tongehalt mit den beschriebenen Gefügemerkmalen verdichtet wurde. - Auffallend sind die z. T. angelösten primären Kalzite, nur ein Teil des Kalkes hat sich unter diesem kalkhaltigen Tschernosem in einen $\mathrm{Ca} / \mathrm{C}$ Horizont mit kleinen Lößkindeln angereichert.

Ein im Hauptprofil durch ca. $3 \mathrm{~m}$ Löß getrennter, blaßbrauner (10 YR 6/3), sch w a ch entwickelter Tschernosem („FF" $\left.\left.{ }_{4 a}{ }^{6}\right)^{6}\right)$ hier bzw. „Mende-F“ nach Pécsi 1959c), wie der untere mit Krotowinen und ähnlichem Gefüge, hat einen noch höheren Kalkgehalt (um 10\%), der zum großen Teil primär sein dürfte, worauf die kaum angelösten Kalzite, fehlende Häufung vom Kalzitmikrolithen in Hohlräumen und Nichtvorhandensein eines $\mathrm{Ca}$-Horizontes hindeuten.

Die im Ziegeleiaufschluß über diesem Boden aufgeschlossenen, von PÉCSI (1965c) als Bodenreste („Mende-E“) bzw. „Semipedolithe“ bezeichnet, waren 1965 anläßlich der Besichtigung durch die Subkommission nur als deutlich geschichtete Bodensedimente anzusprechen.

Über - im Ziegeleiaufschluß - weiteren ca. $7 \mathrm{~m}$ z. T. etwas sandigem Löß sind ähnlich wie bei Paks und anderen ungarischen Lößprofilen (Nogradveröce, Basaharc) zwei Steppenböden aufgeschlossen, deren oberer hier zum größten Teil abgetragen und mit Löß vermischt ist; er wird deshalb im folgenden nicht weiter beschrieben. Der untere besitzt einen besonders auffallend "gefleckten Horizont": (rötlich)braune, scharf umgrenzte, cm-große Flecken in einem dunkelbraunen (7,5 YR 4/2) $80 \mathrm{~cm}$ mächtigen Oberboden, der ebenfalls (etwas) gekappt ist. Im Lößhohlweg ist, wie oben erwähnt, unter dem stärker gekappten, weniger deutlich gefleckten A-Horizont mit verdichtetem Köhärentgefüge ein etwa 15-20 cm mächtiger (B)-Horizont mit einem braunen Farbton (7,5 YR 5/4) und undeutlichem Subpolyedergefüge sichtbar. Mikromorphologisch zeigt der A-Horizont bereits ein relativ dichtes Gefüge. Die Hohlräume sind meist klein und rundlich, ein großer Teil ist mit sekundärem Kalzit mehr oder weniger ausgefüllt. Die Spuren eines einstigen Bodenlebens sind fast überall zu sehen. Die vielen Biotite, meist noch mit deutlichem Pleochroismus lassen an eine nur mäßige Verwitterungsintensität denken. Das Mi-

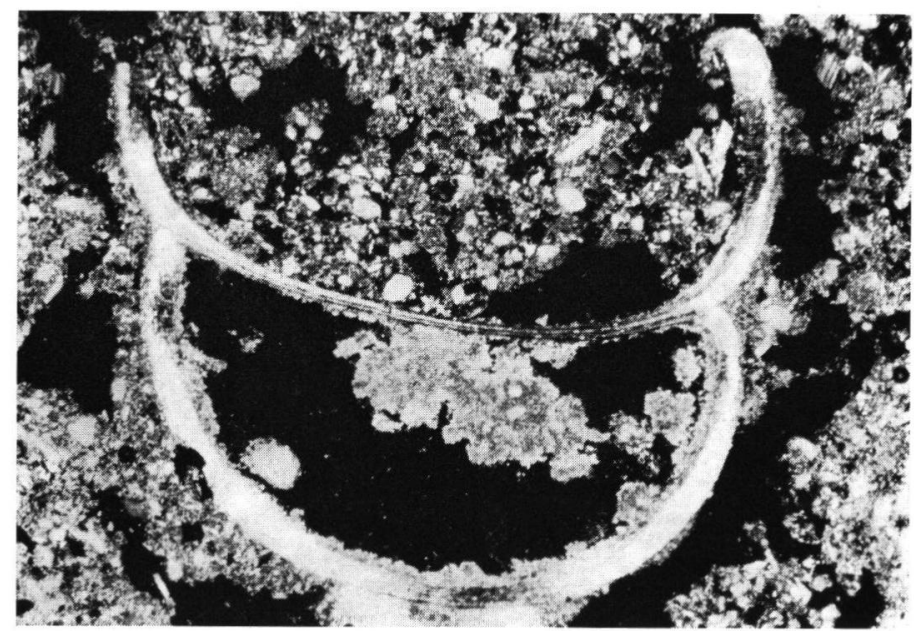

Abb. 7. Löß unter dem "F $\mathrm{F}_{3}$ “-Boden im Lößprofil von Mende. - In Teilen angelöster Conchylienrest, daneben sowohl innerhalb wie außerhalb sekundär ausgefällte Kalzitmikrolithen, Anzeichen für eine hohe Karbonatdynamik. (+ N, Vergrößerung 50-fach.) 
krogefüge im (B)-Horizont ist dem des Oberbodens im ganzen sehr ähnlich, mit häufig größeren Hohlräumen und z. T. schorfigen bis scharfkantig umgrenzten Aggregaten. Es ähnelt mehr einem gut durchflockten Braunerdegefüge, weist aber keine Spuren von Tonverlagerung in Form des Braunlehmteilplasmas auf, was durch die Tongehalte (Tab.1) bestätigt wird.

Der Löß, aus dem dieser B r a u n e r d e - T s cher no s e m ( „F $\mathrm{F}_{3}$ “ hier bzw. „Mende D-Boden“ nach PÉCSI 1965c) hervorging, ist einerseits ein ausgesprochener Ca-Horizont $\left(45 \% \mathrm{CaCO}_{3}\right)$, andererseits ein vorher bereits stärker verwitterter Löß, kenntlich $\mathrm{z}$. B. an stärker angelösten Schneckenschalen (Abb. 7), koprogenen Aggregaten und anderen Organismenspuren (Abb. 8).

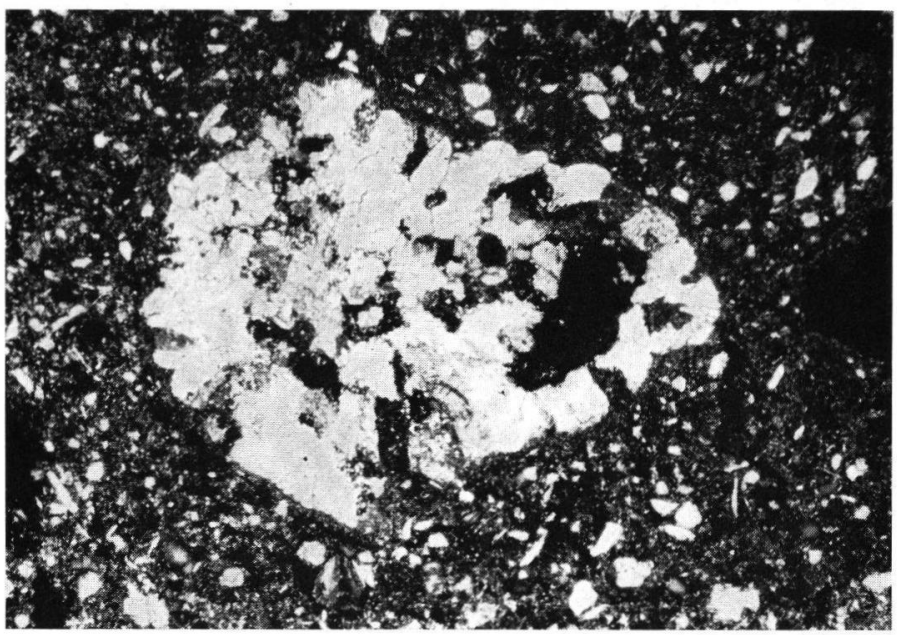

Abb. 8. Gleicher $\mathrm{Ca} / \mathrm{C}-H o r i z o n t$ wie Abb. 7. Ein aus mehreren Kalzitkristallen zusammengewachsenes Kalzitkorn, maximaler Durchmesser 1,2 mm, wahrscheinlich Ausscheidung von Lumbricus terrestris L. ( + N, Vergrößerung 50-fach.)

Die von PÉcsI (1965c) als „Mende B“- bzw. „Mende A“-bezeichneten Böden sind als etwas humose Lösse makroskopisch kaum von Löß zu unterscheiden, viel weniger als z. B. der oberste „F $\mathrm{F}_{1}$ “-Boden im Pakser Profil.

Der basale Boden von Novgradveröce. Der holozäne Boden von Tapiosüly

Über sandig-tonigem Löß, dessen Sandanteil nach unten zunimmt, ist hier an der Basis einer Löß-Boden-Abfolge ein Paläoboden aufgeschlossen, der seinerzeit (1965, vgl. Schlußprotokoll) von vielen Subkommissionsteilnehmern als Pedokomplex (analog dem basalen Bodenkomplex von Mende) ausgesprochen wurde. Über einem ca. $1 \mathrm{~m}$ mächtigen braunen (10 YR 5/3) Unterboden mit Subpolyedergefüge liegt ein $50 \mathrm{~cm}$ mächtiger intensiv dunkelbrauner (10 YR 3/2) Oberboden mit in ausgetrocknetem Zustand dichten Kohärentgefüge direkt auf. An der Grenze zwischen beiden befindet sich ein „gefleckter Horizont“ mit den gleichen Merkmalen, wie sie beim „F $\mathrm{F}_{3}$ “-Boden des Profils von Mende beschrieben wurden. - Schon einige Kalkanalysen lassen die Deutung eines Pedokomplexes unwahrscheinlich erscheinen. Wäre der Oberboden im Sinne eines Bodenkomplexes aus frischem Lößmaterial entstanden, so müßte der Unterboden stärker sekundär aufgekalkt sein (wie im Mende). Der Kalkgehalt beträgt aber nur $1-2 \%$. 
Dagegen ist die sekundäre Aufkalkung des Oberbodens $\left(4,5 \% \mathrm{CaCO}_{3}\right)$, mikromorphologisch kenntlich an den Kalzitmikrolithen in den meist rundlichen, seltener langgestreckten Hohlräumen recht deutlich. Neben rundlichen sind schorfig bis eckig-scharfkantige Aggregate häufiger. In vielen Teilen ist das Gefüge auch hier zwar gut durchflockt aber relativ dicht, damit weniger typisch für einen Tschernosem. Jedoch findet man keine Spur von Braunlehm-Teilplasma wie im Pseudotschernosem (Kubiena 1956).

Auch nach dem mikromorphologischen Befund dürfte die Fleckung wohl auf Humusentfernung in den braunen Stellen (10 YR 5/3) zurückzuführen sein, was RoHDENBURG (1964) an niedersächsischen Böden und Stefanovits (1965) am "Gefleckten Horizont“ in „F $\mathrm{F}_{3}$ “ von Mende nachwiesen. Selbst im Dünnschliff ist eine auffallende Grenze zwischen hell- und dunkelbraunem Material gut sichtbar (Abb. 9).

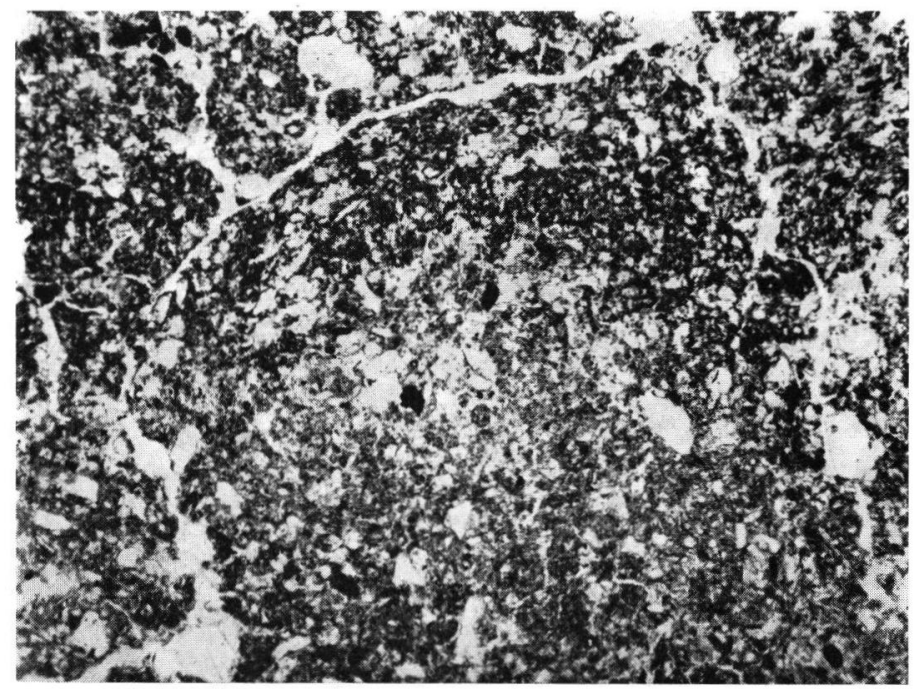

Abb. 9. „Gefleckter Horizont" des basalen Bodens von Nogradveröce. - Die Grenze zwischen hellbraunem (humusärmerem) und dunkelbraunem (humusreicherem) Material ist auch im SchwarzWeiß-Photo noch gut sichtbar. (Hellfeld, Vergrößerung 32-fach.)

Der Unterboden hat ein dichteres aggregatärmeres, im ganzen gut durchflocktes Braunerdegefüge mit etlichen Sprungrissen. Einzelne Teile leiten etwas zu einem erdigen Braunlehmgefüge mit höherer Orientierungsdoppelbrechung über, aber weniger deutlich als im „F5 $\mathrm{F}_{5}$-Boden des Pakser Profils. - Der gut ausgebildete weißliche $\mathrm{CaCO}_{3}$-Horizont (2,5 Y 8/2) mit kleinen Lößkindeln enthält einige Krotowinen mit Unterbodenmaterial. Typologisch entspricht der Boden am ehesten einer (T s chernosem)-B ra u nerde.

Der am weitesten verbreitete Boden des Gödöllö-Monorer Lößhügellandes ist nach Stefanovits und Szücs (1961) ein „Tschernosem-Brauner Waldboden“, in der Nomenklatur von Mückenhausen (1962) ein B ra u n e r d e - T s che rno s e m. Das im Aufschluß von Tapiosüly nahe Mende gelegene Bodenprofil hat unter einem $65 \mathrm{~cm}$ mächtigen dunkelgraubraunen A-Horizont (10 YR 4/2) einen 20-25 cm mächtigen, farblich weniger deutlich abgesetzten braunen (10 YR 5/3) (B)-Horizont, beide sind nahezu kalkfrei $\left(1,4 \% \mathrm{CaCO}_{3}\right)$. Der $\mathrm{Ca} / \mathrm{C}-\mathrm{Horizont}\left(22 \% \mathrm{CaCO}_{3}\right)$ enthält bis faustgroße Lößkindel. Mikromorphologisch hat der A-Horizont ein lockeres, relativ skelettreiches Gefüge und enthält noch mehr große rundliche, wie besonders Mikroaggregate. Aber auch im Unterboden sind die Spuren eines reichen Bodenlebens noch sehr auffällig, häufiger treten pleochroitische Biotite auf. 


\subsection{Untersuchungen $\mathrm{zum}$ Tonmineralbestand 7 )}

Über die mineralogische Zusammensetzung der Fraktion $<2 \mu$ ungarischer Lößböden gibt es einige Untersuchungen mit Hilfe der Differentialthermoanalyse (GEREI 1964, Stefanovits 1959a). Danach scheint in vielen ungarischen Waldböden Illit vorherrschend $\mathrm{zu}$ sein, besonders in solchen, die aus Löß entstanden sind. Jedoch kommt auch Montmorillonit unter den Tonmineralen in Waldböden vor (STEFANovits 1959a, S. 48, zit. aus dem Engl.)

Vom rezenten und fossilen Boden des Pakser Profils liegen ebenfalls DTA-Kurven, jedoch ohne Erläuterungen vor (Stefanovits \& Rozsavölgyi 1962, 148, PÉcsi 1966, 188). Bei den Böden des Lößprofils von Mende unterscheidet Stefanovits (1965) lediglich "tschernosjomartige Tonbildung “ und „unter Wald entstandene Tonminerale“. -

Wie in früheren Arbeiten (Bronger 1966, Bronger, Graf v. Reichenbach \& Schroeder 1966, Bronger 1969b) wurden auch in dieser Arbeit die drei Teilfraktionen Grobton $(2-0,6 \mu)$, Mittelton $(0,6-0,2 \mu)$ und Feinton $(\langle 0,2 \mu)$ getrennt röntgenographisch untersucht. Dadurch wird einerseits das Identifizieren begünstigt, da bestimmte Tonminerale häufig nur in einzelnen Fraktionen vorkommen, wodurch wiederum ein Abschätzen der Mengenverhältnisse erleichtert wird. Eine daraus abgeleitete relativ-quantitative Auswertung ermöglicht dann Aussagen über den Umfang der Neubildung der einzelnen Tonmineralarten in dem fossilen wie in dem rezenten Boden und damit über das Ausmaß der Verwitterung. Diese Schlußfolgerungen werden einmal durch eine annähernde Gleichartigkeit des Ausgangsmaterials (s. u.) sehr erleichtert. Zum anderen haben wir es hier nicht wie in typischen Parabraunerden mit einer ins Gewicht fallenden Tonverlagerung zu tun, wobei auch der ${ } \mathrm{F}_{5}$ “-Boden des Pakser Profils kaum eine Ausnahme macht.

Die Herstellung der Texturpräparate erfolgte nach dem von DümmLer \& Schroeder (1965) beschriebenen Verfahren durch Absaugen der Suspensionen auf poröse keramische Platten. Die dort fixierten Minerale wurden a) mit Magnesium belegt und mit Glycerin gesättigt; zur näheren Diagnostizierung der Tonminerale wurde an den Proben b) eine "feuchte Kaliumbehandlung", c) eine "trockene Kaliumbehandlung", d) eine Hitzebehandlung bei $400^{\circ} \mathrm{C}$ und e) eine Hitzebehandlung bei $550^{\circ} \mathrm{C}$ vorgenommen (DüMmler \& Schroeder, 1965, Bronger, Graf von Reichenbach \& SChroeder 1966, dort nähere Angaben über die Vorbehandlung der Proben). Nach diesen Quell- und Kontraktionstests wurden die Proben in einer Müller-Mikro-101-Apparatur mit Bertholdzählrohrgoniometer ( $\mathrm{CuK} \alpha$-Strahlung, Ni-Filter) untersucht. Bei der Auswertung der ca. 350 Röntgendiagramme wurden insbesondere unterschieden:

1. Montmorillonit, $\mathrm{d}=18 \AA \mathrm{A}$ bei Behandlung a), Kontraktion auf 10 Å bei e)

2. Vermiculit (bzw. aufgeweiteter Illit), $d=14 \AA \AA$ bei a), $10 \AA ̊$ bei c)

3. Illit, $10 \AA$ bei a)

4. Kaolinit, 7,2 Å bei a) bis d), Verschwinden des Reflexes bei e)

5. Chlorit, $14 \AA \AA$ bzw. 7,2 Å bei e)

6. Bodenchlorit, $14 \AA \AA$ bei a) bis c), Verschwinden des Reflexes bei d).

\section{Qualitative Ergebnisse}

Die qualitativen Ergebnisse der Röntgenanalysen der sechs genannten Mineralgruppen sind der Tabelle $1 \mathrm{zu}$ entnehmen. Bei jeder Probe sind die Angaben für Grob-, Mittel- und Feinton aufgeführt.

7) Diese sehr umfangreichen Untersuchungen waren möglich dank der überaus großzügigen Unterstützung des Direktors des Instituts für Pflanzenernährung und Bodenkunde der Universität Kiel, Herrn Prof. Dr. Schroeder. Die Auswertung der Röntgendiagramme wurde gemeinsam mit ihm vorgenommen. 
Tabelle 1:

Korngrößenanalysen und röntgenographische

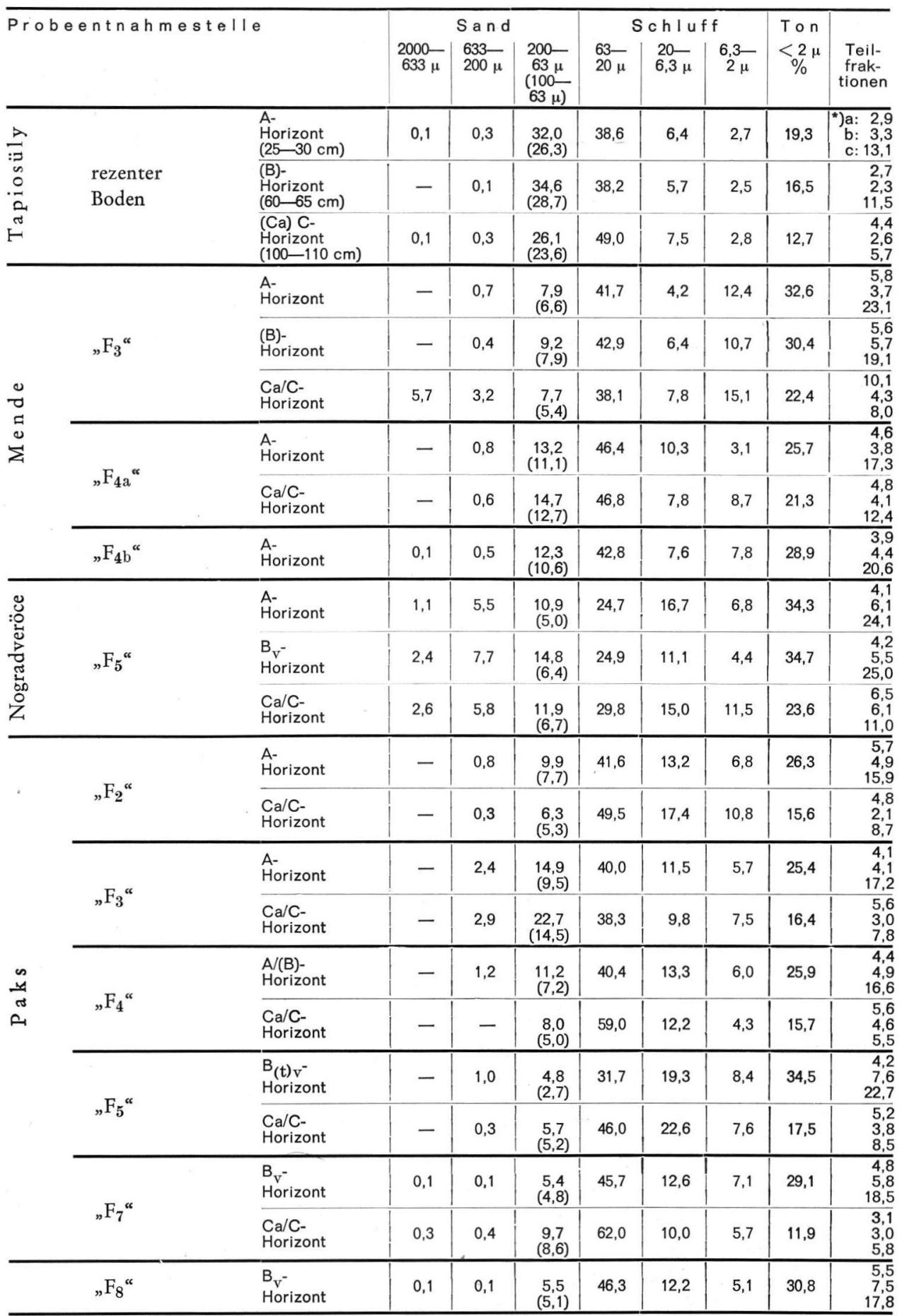

*) $\mathrm{a}=$ Grobton $2-0,6 \mu, \mathrm{b}=$ Mittelton $0,6-0,2 \mu, \mathrm{c}=$ Feinton $\langle 0,2 \mu$. 
Untersuchung der Tonfraktionen

\begin{tabular}{|c|c|c|c|c|}
\hline \multirow[b]{2}{*}{$V \geqq 75 \%$} & \multicolumn{3}{|c|}{$\begin{array}{c}\text { Ton m in era I vertei I u ng } \\
\text { (nach Intensitätsstufen } \vee \text { bis I) }\end{array}$} & \multirow[b]{2}{*}{$15>1 \geqq 5 \%$} \\
\hline & $75>I V \geqq 55$ & $55>111 \geqq 30$ & $30>11 \geqq 15$ & \\
\hline & $M(70)$ & $\begin{array}{l}\text { I(40), V(30), K(30) } \\
\text { I(45), V(30) }\end{array}$ & $\begin{array}{l}\mathrm{K}(25) \\
\mathrm{I}(25)\end{array}$ & $\mathrm{K}(5)$ \\
\hline$M(80)$ & & $\begin{array}{l}V(40), I(30) \\
V(40)\end{array}$ & $\begin{array}{l}\mathrm{K}(25) \\
\mathrm{I}(25), \mathrm{K}(25)\end{array}$ & $\begin{array}{l}C I(5), M(5) \\
C I(5), M(5) \\
I(10), K(10)\end{array}$ \\
\hline$M(90)$ & & $\begin{array}{l}K(35), I(30) \\
M(50)\end{array}$ & $\begin{array}{l}\mathrm{V}(20) \\
\mathrm{I}(25), \mathrm{K}(20)\end{array}$ & $\begin{array}{l}\mathrm{M}(10), \mathrm{Cl}(5) \\
\mathrm{Cl}(5) \\
\mathrm{I}(5), \mathrm{K}(5)\end{array}$ \\
\hline \multirow[t]{6}{*}{$M(80)$} & & $\begin{array}{l}\mathrm{I}(35), \mathrm{K}(35), \mathrm{Cl}(30) \\
\mathrm{K}(35), \mathrm{Cl}(35)\end{array}$ & $\begin{array}{l}\mathrm{I}(25) \\
\mathrm{I}(15)\end{array}$ & $\begin{array}{l}M(5) \\
K(5)\end{array}$ \\
\hline & $M(55)$ & $\begin{array}{l}\mathrm{K}(40), \mathrm{Cl}(35) \\
\mathrm{M}(30), \mathrm{K}(30) \\
\mathrm{I}(40)\end{array}$ & $\begin{array}{l}I(25) \\
I(20), C I(20)\end{array}$ & $\mathrm{K}(5)$ \\
\hline & & $\begin{array}{l}\text { I(35), CI(35), K(30) } \\
\text { M(30), K(30) } \\
\text { I(50), M(45) }\end{array}$ & $\mathrm{I}(20), \mathrm{CI}(20)$ & $K(5)$ \\
\hline & $M(55)$ & $\begin{array}{l}\mathrm{I}(35, \mathrm{Cl}(35), \mathrm{K}(30) \\
\mathrm{I}(40)\end{array}$ & $\mathrm{M}, \mathrm{I}, \mathrm{K}, \mathrm{Cl}$ (je 25) & $\mathrm{K}(5)$ \\
\hline & $M(55)$ & $\begin{array}{l}J(35), C I(35), K(30) \\
I(50), M(45)\end{array}$ & I, K, CI (je 15) & $K(5)$ \\
\hline & $M(60)$ & $\begin{array}{l}\mathrm{Cl}(40), \mathrm{I}(30), \mathrm{K}(30) \\
\mathrm{I}(30), \mathrm{K}(30) \\
\mathrm{I}(35)\end{array}$ & $\mathrm{Cl}(20), \mathrm{M}(20)$ & $\mathrm{K}(5)$ \\
\hline$M(80)$ & & $\begin{array}{l}\mathrm{V}(40), \mathrm{I}(40) \\
\mathrm{I}(50)\end{array}$ & $\begin{array}{l}\mathrm{K}(15), \mathrm{K}(15), \mathrm{ClB}(15) \\
\mathrm{V}(20), \mathrm{K}(15), \mathrm{B}\end{array}$ & $\begin{array}{l}\mathrm{Cl}_{\mathrm{B}}(5) \\
\mathrm{K}(5)\end{array}$ \\
\hline$M(80)$ & & $\begin{array}{l}V(40), I(30) \\
M(30), I(30), K(30)\end{array}$ & $\begin{array}{l}\mathrm{K}(20) \\
\mathrm{I}(15)\end{array}$ & $\begin{array}{l}\mathrm{Cl}(5), \mathrm{Cl}(5) \\
\mathrm{V}(10) \\
\mathrm{K}(5)\end{array}$ \\
\hline & $\begin{array}{l}M(65) \\
M(70) \\
\end{array}$ & & $\begin{array}{l}1(25), K(25), M(20), V(20) \\
I(15)) \\
I(20)\end{array}$ & $\begin{array}{l}\mathrm{Cl}(10) \\
\mathrm{K}(10), \mathrm{CI}(10) \\
\mathrm{K}(10)\end{array}$ \\
\hline$M(90)$ & & $\begin{array}{l}\mathrm{K}(35) \\
\mathrm{M}(40)\end{array}$ & $\begin{array}{l}\mathrm{I}(25), \mathrm{M}(20), \mathrm{Cl}(20) \\
\mathrm{I}(25), \mathrm{K}(25)\end{array}$ & $\begin{array}{l}\mathrm{Cl}(10) \\
\mathrm{I}(5), \mathrm{Cl}(5)\end{array}$ \\
\hline$M(80)$ & & $\begin{array}{l}\text { I(40), K(30) } \\
\text { I(40), K(30) } \\
\end{array}$ & $\begin{array}{l}\mathrm{Cl}(25) \\
\mathrm{Cl}(25)\end{array}$ & $\begin{array}{l}M(5) \\
M(5), C l(5), K(5) \\
I(10),\end{array}$ \\
\hline$M(80)$ & & $\begin{array}{l}\mathrm{I}(40), \mathrm{K}(30), \mathrm{Cl}(30) \\
\mathrm{I}(30), \mathrm{K}(30)\end{array}$ & $\begin{array}{l}\mathrm{Cl}(15) \\
\mathrm{I}(15)\end{array}$ & $\begin{array}{l}\mathrm{M}(5) \\
\mathrm{K}(5)\end{array}$ \\
\hline \multirow[t]{2}{*}{$M(80)$} & & $\begin{array}{l}\mathrm{K}(35), \mathrm{I}(30), \mathrm{Cl}(30) \\
\mathrm{K}(40), \mathrm{I}(30)\end{array}$ & $\mathrm{Cl}(20)$ & $\begin{array}{l}M(5) \\
M(10) \\
M(10), K(5), C l(5)\end{array}$ \\
\hline & $M(70)$ & $\begin{array}{l}I(40), K(30), \mathrm{Cl}(30) \\
\text { I(45), } \mathrm{K}(30)\end{array}$ & $\begin{array}{l}\mathrm{Cl}(15) \\
\mathrm{I}(25)\end{array}$ & $\begin{array}{l}M(10) \\
K(5)\end{array}$ \\
\hline$M(80)$ & & $\begin{array}{l}I(30), K(30) \\
M(50)\end{array}$ & $\begin{array}{l}\mathrm{M}(20), \mathrm{Cl}(20) \\
I(20), \mathrm{K}(20) \\
\mathrm{I}(20)\end{array}$ & $\mathrm{Cl}(10)$ \\
\hline$M(90)$ & & $\begin{array}{l}\text { I(30), K(30) } \\
\text { I(45) }\end{array}$ & $\begin{array}{l}\mathrm{CI}(20), \mathrm{V}(20) \\
\mathrm{K}(25), \mathrm{M}(20)\end{array}$ & $\begin{array}{l}C I(10) \\
I(10)\end{array}$ \\
\hline$M(80)$ & $M(60)$ & $\mathrm{I}(35), \mathrm{K}(30)$ & $\begin{array}{l}\mathrm{M}(20), \mathrm{Cl}(15) \\
\mathrm{I}(20) \\
\mathrm{I}(20)\end{array}$ & $\mathrm{K}(10), \mathrm{CI}(10)$ \\
\hline$M(90)$ & & $\begin{array}{l}(30), \mathrm{Cl}(30), \mathrm{K}(30) \\
\mathrm{I}(45)\end{array}$ & $\mathrm{K}(25), \mathrm{M}(15), \mathrm{Cl}(15)$ & $\begin{array}{l}\mathrm{V}(10) \\
\mathrm{I}(10)\end{array}$ \\
\hline $\mathrm{M}(80)$ & $M(70)$ & $M(30), I(30)$ & $\begin{array}{l}\mathrm{K}(25), \mathrm{CI}(15) \\
\mathrm{I}(15), \mathrm{Cl}(15) \\
\mathrm{I}(20)\end{array}$ & \\
\hline$M(80)$ & & $\begin{array}{l}\mathrm{I}(40), \mathrm{K}(30) \\
M(30), \mathrm{I}(30), \mathrm{K}(30)\end{array}$ & $\begin{array}{l}\mathrm{Cl}(20) \\
\mathrm{I}(20)\end{array}$ & $\begin{array}{l}\mathrm{V}(10) \\
\mathrm{Cl}(10)\end{array}$ \\
\hline
\end{tabular}

$\mathrm{M}=$ Montmorillonit, $\mathrm{V}=$ Vermikulit, $\mathrm{I}=\mathrm{Illit}, \mathrm{K}=$ Kaolinit, $\mathrm{Cl}=$ Chlorit, $\mathrm{Cl}_{\mathrm{B}}=$ Bodenchlorit. 
In allen Proben - Lösse, pleistozänen Böden recht unterschiedlicher Genese und holozäner Böden - sind Montmorillonit, Illit und Kaolinit vorhanden. Dabei findet sich Illit stets in allen drei Teilfraktionen, Kaolinit stets im Grobton, fast immer im Mittelton und bei $2 / 3$ aller Proben im Feinton. Montmorillonit tritt stets in der Feintonfraktion auf, fast immer auch im Mittelton. In den Lössen ist Montmorillonit meistens (in 7 von 9 Fällen) auch im Grobton vorhanden; in den Böden fehlt er dagegen, von einer Ausnahme abgesehen.

Ein auffallender Unterschied zum Tonmineralbestand südbadischer Lößprofile (BRONGER 1966, 1969b) besteht darin, daß in den hier untersuchten ungarischen Lößprofilen Chlorit ebenfalls in allen Proben im Grob- und Mittelton, gelegentlich sogar im Feinton vorkommt. Im A- und $\mathrm{B}_{\mathrm{v}}$-Horizont des basalen Bodens in Nogradveröce finden sich daneben Bodenchlorite. Vermiculite sind im rezenten Boden und im basalen Boden in Nogradveröce sowie den jeweils unterlagernden Lössen, außerdem in den drei unteren noch erhaltenen B-Horizonten des Pakser Profils stets in der Grobtonfraktion, seltener im Mittelton, nie im Feinton zu beobachten. Minerale mit Wechsellagerungsstruktur, kenntlich an breiteren Interferenzen zwischen 10 und $14 \AA$ A, sind (entsprechend früheren Untersuchungen) nur gelegentlich auf den Diagrammen angedeutet, so daß sie in die Tabelle nicht aufgenommen wurden.

Darüber hinaus tritt im Grobton a 11 e r Proben Quarz in einem meist sehr starken Reflex von $\mathrm{d}=3,35 \AA$ und einem deutlichen von 4,25 $\AA$ in Erscheinung, weniger im Mittelton und selten in der Feintonfraktion. Hingegen konnten Feldspäte, kenntlich an den Interferenzen von $\mathrm{d}=3,25 \AA$ und $3,20 \AA$, selbst im Grobton kaum nachgewiesen werden.

Beim Vergleich des Tonmineralbestandes der Lösse mit dem der jeweils aus ihnen entstandenen Böden lassen sich — von den erwähnten Ausnahmen abgesehen - quali t a t iv kaum Unterschiede feststellen. Im Ganzen weichen die elf quartären Böden sieht man von den häufig fehlenden Vermiculiten bzw. den nur in einem Boden (s.o.) auftretenden Bodenchloriten $\mathrm{ab}$ - im qualitativen Tonmineralbestand nur wenig voneinander $a b$, trotz ihrer recht verschiedenen Genese und, soweit bereits mikromorphologisch feststellbar, unterschiedlichen Verwitterungsintensität.

Wie schon bei der Untersuchung von Böden südbadischer Lößprofile festgestellt wurde, gibt auch hier die qualitative Zusammensetzung des Tonmineralbestandes keine Aufschlüsse über eine verschieden hohe Intensität der Verwitterung und das Ausmaß der Bodenbildung. Dieses Fazit unterstreicht erneut die Notwendigkeit des Versuchs einer relativquantitativen Auswertung, um zu einer angenäherten Vorstellung über den Umfang der Neubildung der einzelnen Tonmineralarten zu kommen.

\section{Relativ-quantitative Auswertung}

Wie schon früher (Bronger 1966a, b, 1969b) wurde angesichts der Problematik einer quantitativen Tonmineralbestimmung (u. a. DüMmLer \& Schroeder 1965) der Weg einer relativ-quantitativen Abschätzung des Tonmineralbestandes gewählt. Die verhältnismäßig einfache Zusammensetzung der Proben ermöglicht zumindest in Annäherung eine Vorstellung über die Mengenanteile, besonders der oben genannten fünf Mineralgruppen (Bodenchlorite kommen nur in zwei Proben vor). Durch den Vergleich der Intensitäten der Basisreflexe $>7 \AA$, insbesondere aus den Intensitätsveränderungen des $10 \AA \AA$-Reflexes nach den Behandlungen b), c) und e) (Dümmler \& Schroeder 1965, 44, Bronger 1969b, Abb. 12 u. 13) wurden die Anteile in Prozenten der Summe der Montmorillonit-VermiculitIllit-Kaolinit-Chlorit-Bodenchlorit-Gehalte ausgedrückt (Abb. 10). Die auf diese Weise erhaltenen Zahlen sollen nicht als absolute Zahlen betrachtet werden, sondern nur eine Größenordnung angeben. Deshalb wurden die Prozentwerte zu fünf Gruppen bzw. fünf Intensitätsstufen I-V zusammengefaßt. In Tabelle 1 sind die Minerale nach ihren Basis- 

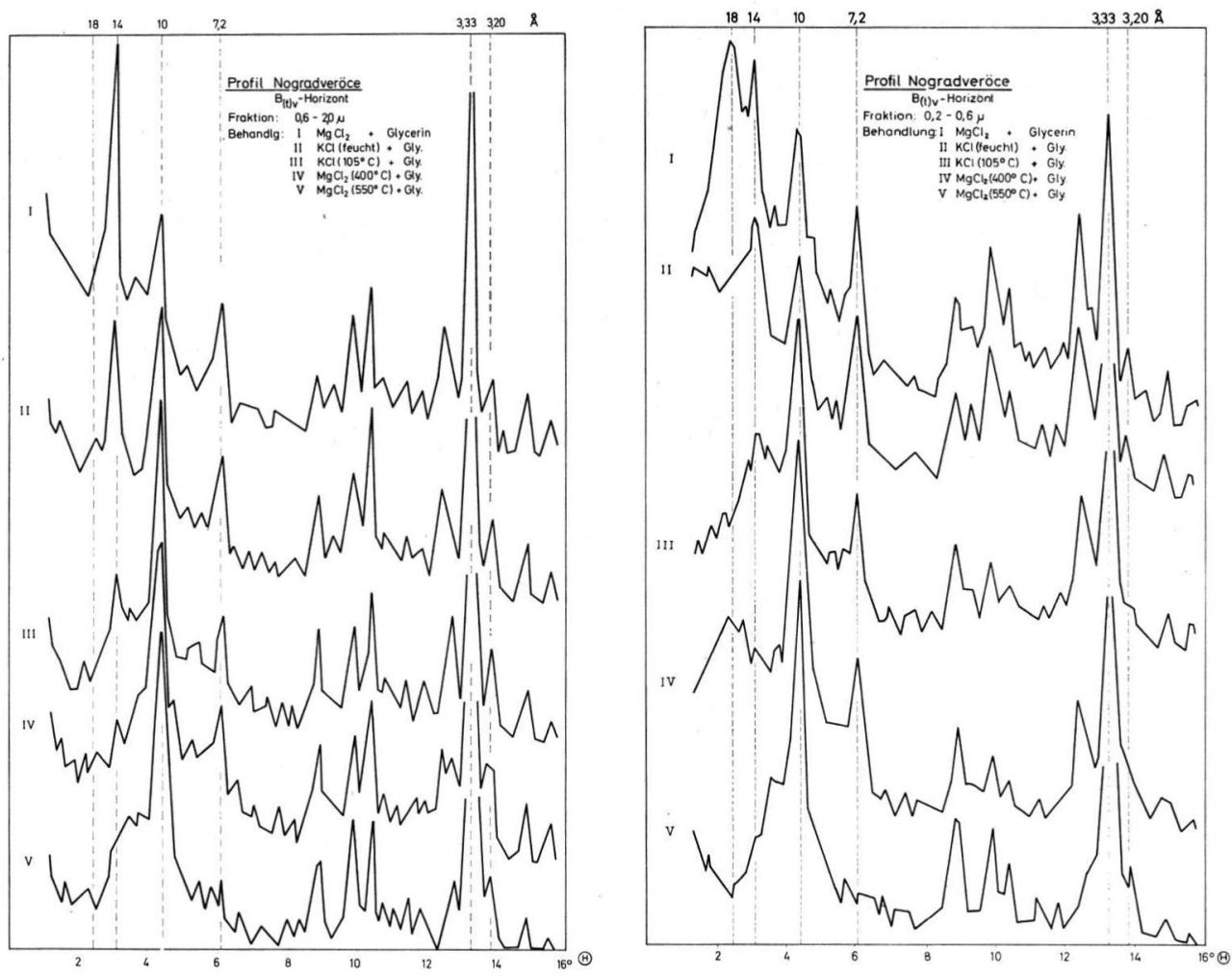

Abb. 10. Röntgendiagramme von verschieden behandelten Texturpräparaten der Grob- (a) und Mitteltonfraktion (b) vom $\mathrm{B}_{(t) v}$-Horizont des basalen Bodens im Lößprofil von Nogradveröce. Hier verringert sich im Grobton der $14 \AA$-Reflex nach der "feuchten Kaliumbehandlung" infolge beträchtlicher Anteile von Vermiculit, während sich der $10 \AA$-Reflex entsprechend verstärkt. Der Rest des $14 \AA$-Reflexes weist auf sehr geringe Anteile von Bodenchlorit (Abnahme nach Behandlung d) und Chlorit hin. - Im Mittelton bedeutet die Zunahme des $10 \AA$-Reflexes nach der Behandlung c.) bzw. e.) geringe Anteile von Vermiculit und beträchtliche Anteile von Montmorillonit.

reflex-Intensitäten in eine entsprechende fünfstufige Skala eingeordnet. Durch Multiplikation der Intensitätsstufen V-I der jeweiligen Tonminerale mit den entsprechenden Anteilen der Tonfraktion (Tabelle 1, linke Seite) und Darstellung der erhaltenen Werte als Säulendiagramme (Abb. 1 und 2) erhält man ein Bild über das Ausmaß der Neubildung der einzelnen Tonmineralarten in den Böden.

$\mathrm{Da}$ in die berechneten Werte die Anteile der Tonfraktionen mit eingehen, sei zunächst auf die Unterschiede im Gesamttongehalt (Fraktion $<2 \mu$ ) sowie die Unterschiede in den Gehalten an Grob-, Mittel- und Feinton eingegangen. Neben der Darstellung der Analysendaten in der entsprechenden Spalte in den Abb. 1 und 2, sind die Werte auch der gröberen Fraktionen der nichtkarbonatischen Substanz (entkalkt bei $\mathrm{pH}>4$ ) in Tab. 1 angegeben.

Aus den Daten folgt einmal, daß - im Unterschied zu den untersuchten Lössen des Oberrheingebietes (1966a, b, 1969b) die Lösse in der Mehrzahl einen noch mäßigen Sandanteil haben. Die Lösse mit stärkerer Sandbeimengung (Nogradveröce, Löß des „ $\mathrm{F}_{3}$ “ in Paks) haben zugleich aber auch einen relativ hohen Tongehalt. Dennoch darf auch hier von der Korngrößenverteilung her von einer weitgehenden sedimentären Homogenität zwischen den Lössen und den daraus entstandenen Böden gesprochen werden; auch die Abweichung im Falle des „ $\mathrm{F}_{\mathbf{3}}$ “ im Pakser Profil ist nicht zu groß. 
Bemerkenswert ist zunächst der nicht geringe Grad der „Verlehmung“ der oberen drei mehr oder weniger degradierten bis verbraunten Tschernoseme des Lößprofils von Paks und entsprechend des ${ } \mathrm{F}_{3}$ " und des ${ }^{\circ} \mathrm{F}_{4} \mathrm{~b}$ " in Mende (Abb. 2), d.h. die Zunahme der Tonfraktion8) um 9-12\% gegenüber der der jeweils unterlagernden Lösse. Diese Verlehmung drückt sich insbesondere in der Zunahme der Feintonfraktion aus, ohne daß hier eine nennenswerte Verlagerung dieser Fraktion wie bei Löß-Parabraunerden $(1966,1969 \mathrm{~b})$ in Frage kommt. Die Vermehrung des Tonanteils erreicht die des basalen Bodens von Nogradveröce und übertrifft die des rezenten Braunerde-Tschernosems, der freilich einen beträchtlichen Feinsandanteil aufweist. Es ist aber notwendig, mehrere rezente Lößböden in einen solchen Vergleich mit einzubeziehen. Entsprechende Profile sind in Arbeit.

Noch deutlich höher ist der Grad der Verlehmung in den unteren drei Waldböden des Profils von Paks. ${ }^{9}$ ) Ebenfalls ist hier die Tonzunahme größtenteils in der Feintonfraktion zu beobachten, die dann bis zu $2 / 3$ der Gesamttonfraktion ausmacht. Auch in diesen Waldböden fällt eine Erhöhung gerade der Feintonfraktion durch Verlagerung nicht ins Gewicht.

Bei vorsichtiger Auswertung der Tonmineralgehalte in den drei Tonfraktionen der verschiedenen Löß- und Bodenproben (Tab. 1, Abb. 1 und 2) seien folgende Ergebnisse hervorgehoben:

1. Auch die hier untersuchten Lösse recht verschiedenen Alters zeigen einmal einen merklichen Kaolinitgehalt, zum anderen einen oft hohen Montmorillonitgehalt, besonders in der Feintonfraktion, so daß er insgesamt den der Illite übertreffen kann. Dieser Befund entspricht durchaus denjenigen früherer Untersuchungen (BRONGER 1966, 1969b) an südbadischen Lössen.

2. Beim Vergleich des Tonmineralbestandes der Tonfraktionen der Lösse mit denen der aus ihnen entstandenen Böden sowie der Böden untereinander sind gegenüber Lößparabraunerden Südbadens ${ }^{10}$ ), wo sich eine deutliche bis starke Zunahme a ller Mineralkomponenten (deutlich beim Kaolinit, etwa gleichermaßen stark beim Illit und Montmorillonit) zeigte, bemerkenswerte Unterschiede festzustellen:

a) In allen Böden (mit Ausnahme des schwach entwickelten Tschernosems — „ $\mathrm{F}_{4 \mathrm{a}}{ }{ }$ in Mende) überwiegt die Neubildung des Montmorillonits bei weitem. Sie ist zwar auch hier fast ausschließlich auf die Feintonfraktion beschränkt, jedoch sind die hohen Montmorillonitanteile auch in den B-Horizonten höchstens zu einem geringen Teil auf Verlagerung zurückzuführen. Die Zunahme ist in den Waldböden (soweit feststellbar) im Pakser Profil deutlich höher als in den hangenden Waldsteppenböden ( $\mathrm{F}_{4}$ “ $-{ } \mathrm{F}_{2}$ “) ; im Braunerde-Tschernosem $\left({ } \mathrm{F}_{3}\right.$ “) des Profils von Mende jedoch (mitbedingt durch den hier besonders hohen Feintonanteil) ist sie ebenfalls beträchtlich und bedeutend höher als im rezenten Boden.

b) Die Illitneubildung tritt vergleichsweise stärker zurück, besonders in den Böden des Pakser Profils. Eine Neubildung von Kaolinit ist nur in einigen Böden recht gering bzw. in Spuren feststellbar. Vergleichsweise recht schwach (gegenüber den südbadischen Waldböden) ${ }^{10}$ ) ist auch die Neubildung von Vermiculiten im basalen Boden von Nogradveröce, im " $\mathrm{F}_{5}$ “ und „ $\mathrm{F}_{8}$ “ von Paks bzw. im rezenten Boden. Diese geringe oder fehlende Vermiculitneubildung deutet andererseits darauf

8) Von den Böden des Lößprofils von Mende existieren bereits Korngrößenanalysen (STEFANovirs 1965), jedoch wurde der jeweils zugehörige C-Horizont anscheinend $\mathrm{n} \mathrm{i} \mathrm{cht}$ mituntersucht. Aussage über das unterschiedliche Ausmaß der Tonbildung (a.a. O., S. 342) in den einzelnen Böden bleiben daher unklar. - Obwohl Kriván seine Proben wohl weiter im Norden des Pakser Profils nahm, sind seine Korngrößenangaben (1955b, Tabelle im Anhang) mit oft geringerem Tongehalt in den fossilen Böden, wo selten über $10 \%$ ermittelt wurde als in den unterlagernden Lössen, nicht ohne weiteres erklärlich.

9) Die Tonzunahme des basalen Bodens im Lößprofil von Mende ist etwa ebenso hoch, wobei hier aber (s. o.) Tonverlagerung in Form des Braunlehm-Teilplasmas zu beobachten ist.

10) Aus Gründen des veränderten Maßstabes konnten hier in den Abb. 1 und 2 - gegenüber entsprechenden Säulendiagrammen südbadischer Lößprofile - die Säulen bei entsprechender Breite jeweils nur mit halber Höhe dargestellt werden, was man bei einem Vergleich beachten mag. 
hin, daß besonders die Montmorillonitbildung nicht nur wie die Illitbildung durch teilweisen Abbau und Umwandlung von Glimmern, sondern auch durch völligen Abbau von Feldspäten und nachfolgender Synthese aus den Zerfallsprodukten entstanden sein könnte. Besonders in den Waldsteppenböden sind in den gröberen Fraktionen im Dünnschliff etliche mehr oder weniger angewitterte Feldspäte zu beobachten, während sie bereits im Grobton - im Gegensatz zu den deutlichen Quarzanteilen - kaum nachzuweisen sind, was im Widerspruch zu den Ergebnissen anderer Autoren (Rohdenburg \& Meyer 1966, Scheffer at al. 1966) steht. Jedoch soll dieses spezielle Problem der Feldspatverwitterung in einer gesonderten Arbeit näher untersucht werden.

\section{Schlußfolgerungen und Diskussion}

Vergleicht man in den hier untersuchten Lößböden den jeweiligen Umfang der Neubildung der einzelnen Tonmineralarten als ein Ausdruck der Intensität ihrer Verwitterung, so kann man feststellen, daß hierin die Waldsteppenböden ( „ $\mathrm{F}_{4}$ “ bis „ $\mathrm{F}_{2}$ “) den unteren drei Waldböden des Pakser Profils nicht viel nachstehen. Andererseits ist das Ausmaß der Tonmineralneubildung in den drei „ $\mathrm{F}_{4}$ “- bis „ $\mathrm{F}_{2}$ “-Böden in Paks bzw. den beiden „ $\mathrm{F}_{4 \mathrm{~b}}$ “ bzw. „ $\mathrm{F}_{3}$ “-Böden in Mende gleich groß bzw. größer als beim typologisch vergleichbaren rezenten Boden. Hier müssen freilich noch weitere rezente Böden ähnlicher Genese in die vergleichende Untersuchung mit einbezogen werden.

Besonders die Löß-Boden-Abfolge des Pakser Profils vom jüngsten fossilen Waldboden an aufwärts, wie sie in Abb. 1 dargestellt ist, entspricht weitgehend derjenigen von mehreren großen Lößprofilen im südlichen Karpatenbecken, so daß hier von einer Standard-Abfolge gesprochen werden kann. Lediglich in Mende und im Lößprofil von Basaharc nahe Esztergom ist der in Paks als " $\mathrm{F}_{4}$ " bezeichnete Boden nochmals unterteilt11), wobei aber jeweils der oberste Boden $\left({ }^{2} \mathrm{~F}_{4 a}\right.$ ") in beiden Fällen nur durch eine recht schwache Verwitterung gekennzeichnet ist. - Der Fauneninhalt der meisten Lößabschnitte, besonders der oberen $20 \mathrm{~m}$ des Pakser Profils (HoRváth 1954) ist dem von nördlicher gelegenen Lößgebieten Mitteleuropas (LožEK 1965) ähnlich.

Wie am Anfang (S. 124/125) erwähnt, hielten alle Bearbeiter von Bulla bis Kriván die in Paks sichtbare bzw. früher sichtbare Schichtenfolge für eine komplette Serie des Pleistozäns, wobei nur KrIVÁN (1955b) ein Fehlen der Günzablagerungen annahm. Nach vergleichenden Untersuchungen an Lößprofilen des Karpatenbeckens (BRONGER 1969c) ist die Löß-Boden-Abfolge in Paks nicht annähernd vollständig. So ist im Lößprofil von Stari Slankamen Čot (gegenüber der Mündung der Theiß in die Donau) zwischen den stratigraphisch äquivalenten Böden ${ } \mathrm{F}_{5}$ “ bis ${ } \mathrm{F}_{2}$ " und der zwei liegenden Braunerden hier wie dort ein typologisch andersartiger, intensiv rötlich gefärbter, nach dem mikromorphologischen Befund etwas rubefizierter erdiger Braunlehm-(Lessivé) aufgeschlossen, mit viel höherer Verwitterungsintensität als in allen Paläoböden von Paks. In Liegenden der beiden Braunerden in Paks $\left({ } \mathrm{F}_{7}\right.$ " und ${ } \mathrm{F}_{8}$ “, die sicher kein praepleistozänes Alter aufweisen, wie Kriván 1955b und FINK 1965, S. 273 meinen), war früher noch ein rötlich brauner Boden aufgeschlossen (AdAM, Marosi \& SzILÁrd 1954). In Slankamen folgen unter den (aller Wahrscheinlichkeit nach zeitlich äquivalenten) beiden Braunerden weitere drei stark rot gefärbte, intensiv verwitterte, erdige Braunlehme (BRONGER 1969c).

Für eine abschließende paläoklimatische Auswertung unserer Untersuchungsergebnisse muß auf die viel diskutierte Frage kurz eingegangen werden, welcher der Böden etwa des Pakser Profils das Riß/Würm-Interglazial repräsentiert. Bulla (1934, 1937), Scherf (1936) und Adam, Marosi \& SzilláRd (1954) hielten den hier als „F “ " bezeichneten Boden für das Riß/Würm-Interglazial, PÉCSI (1966) den „F $\mathrm{F}_{5}$ “-Boden. Mehrere Teilnehmer der o.g. Tagung der Subkommission für Löß-Stratigraphie vermuteten es besonders wegen des (B)-Horizontes im " $\mathrm{F}_{3}$ “-Boden von Mende. Dieser Boden entspricht einerseits trotz seiner an dieser Stelle intensiveren Ausbildung dem „ $\mathrm{F}_{3}$ “-Boden von Paks und ist andererseits dem "gefleckten Horizont" des (am loc. typ., stärker erodierten) „Paudorfer Bodens“ typologisch ähnlich, ohne daß er ihm stratigraphisch genau entsprechen müßte. Außerdem existiert eine ${ }^{14} \mathrm{C}$-Analyse von Kohleresten im Bodenrest des „ $\mathrm{F}_{2}$ “-Bodens in Mende, die

11) In Dellenpositionen kann der eine oder andere Boden gedoppelt auftreten. 
ein Alter von $29800 \pm 600$ Jahre ergab (PÉCSI 1965); freilich sind weitere ${ }^{14}$ C-Bestimmungen notwendig. Besonders im Hinblick auf den häufig zitierten „stratigraphischen Leitsatz “, daß der jüngste fossile Waldboden das letzte Interglazial repräsentiert (FINK 1962, 19), ist die Einordnung, wie PÉcsı sie annimmt, am wahrscheinlichsten. Die beiden Böden ${ } \mathrm{F}_{3}$ “ und ${ } \mathrm{F}_{2}$ “ würden dann stratigraphisch am ehesten den Interstadialen von Hengelo und Denekamp entsprechen, der „ $\mathrm{F}_{4}$ “-Boden bzw. die „ $\mathrm{F}_{4 \mathrm{a}}$ “ und „ $\mathrm{F}_{4 \mathrm{~b}}$ “-Böden vielleicht den Interstadialen von Amersfoort-Brörup. ${ }^{12}$ )

Aber die Frage nach einem letztinterglazialen Boden setzt in diesem Gebiet unrichtige Akzente. Aus den Untersuchungen hat sich vor allem ergeben, daß in diesem Raum für die jungpleistozänen Böden ( $\mathrm{F}_{4}$ “ bis „ $\mathrm{F}_{2}$ “) eine Unterscheidung nach Interstadial- oder Interglazialcharakter keinen Sinn hat. Auch diese Böden sind - im Gegensatz zu den zeitlich entsprechenden in Nordwest-Mitteleuropa (vgl. u. a. Zagwijn und PAEPE 1968) - Bildungen eines warmzeitlichen Klimas, da sie bei ähnlicher typologischer Ausbildung ihrem Verwitterungsgrad nach den des heutigen Klimaxbodens dieser Gebiete mindestens erreichen. Manche oft heftig geführte Diskussionen um den „letztinterglazialen Boden“ im südöstlichen Mitteleuropa erscheinen bei Berücksichtigung dieses Sachverhalts als teilweise aneinander vorbeigeführt. -

\section{Literatur}

Adam, L., Marosi, S. \& Szilárd, J.: A paksi löszfeltárás. (Der Lößaufschluß von Paks). Földrajzi Közlemények 78, 2, 239-254, Budapest 1954.

Bacsák, Gy.: A pliozen és a pleistocén az égi mechanika in egvilági tásában. (Das Pliozän und Pleistozän im Lichte der Himmelsmechanik). - Földtáni Közlöny, 3, 70-105, Budapest 1955.

Bronger, A.: Lösse, ihre Verbraunungszonen und fossilen Böden. Ein Beitrag zur Stratigraphie des oberen Pleistozäns in Südbaden. - Schriften des Geogr. Inst. d. Univ. Kiel, XXXIV, H. 2, 104 S., Kiel 1966 (1966a).

- : Zur Klimageschichte des Quartärs von Südbaden auf bodengeographischer Grundlage. Peterm. Geogr. Mitt., 113, 2, 112-124, Gotha 1969 (1969a).

- : Zur Mikromorphogenese und zum Tonmineralbestand quartärer Lößböden in Südbaden. Geoderma, 3 (1969/1970), Amsterdam 1970.

- : Zur Mikromorphologie und Genese von Paläoböden aus Löß im Karpatenbecken. - Third Intern. Working Meeting on Soil Micromorpholgy, Wroclaw 1969 (1969c) (im Druck).

Bronger, A. Graf v. Reichenbach, H. \& Schroeder, D.: Über den Tonmineralbestand des Lößprofils von Heitersheim, Südbaden. - Z. Pflanzenernähr., Düng., Bodenkunde, 113, 3, 193-203, Weinheim 1966 (1966b).

Bulla, B.: A magyarországi löszög és folyóterraszok problémái. (Zum Problem der ungarischen Lösse und Flußterrassen). - Földrajzi Közlemények, LXII k., 136-149, 166-168, Budapest 1934.

— : Der pleistozäne Löß im Karpatenbecken. - Földtáni Közlöny, 196-215, 289-309, 1937; 33-58, Budapest 1938.

Diskussionsmaterial und Protokoll der Schluß-Sitzung anläßlich der Tagung der Subkommission für Lößstratigraphie der INQUA, Budapest. - Földrajzi Közlemények, 89, 371-384, Budapest 1965.

DümmleR, H. \& SChroeder, D.: Zur qualitativen und quantitativen röntgenographischen Bestimmung von Dreischicht-Tonmineralen in Böden. - Z. Pflanzenernähr., Düng., Bodenkunde, 109, 1, 35-47, Weinheim 1965.

Exkursionsführer, I. Bd., I. und III. Exkursion. - VIII. Intern. Bodenkundl. Kongreß, 385 S., Bukarest 1964.

Fink, J.: Die Gliederung des Jungpleistozäns in Österreich. - Mitt. d. Geol. Ges. in Wien, 54, 1 -25, Wien 1962.

- Die Subkommission für Lößstratigraphie der Internationalen Quartärvereinigung. - Eiszeitalter u. Gegenwart, 16, 264-275, Öhringen 1965.

12) Von bodengeographischen Uberlegungen her ist es eher denkbar, daß der „Paudorfer Boden“ als Tschernosem einem der Tschernoseme (i. w. S.) des „PK II“ tschechischer Nomenklatur auch stratigraphisch entspricht; der braune Verwitterungshorizont (schwach entwickelte Braunerde?) des "Stillfried-B-Bodens“ eher dem „PK I" der tschechischen Profile, was ähnlich auch schon Rohdenburg \& Meyer (1966) annahmen. 
GEREI, L.: Mikromineralogische Zusammensetzung einiger ungarischer Bodentypen (Zusammenfassung). - Agrokémia és Talajtan, 13, 269, Budapest 1964.

Honváth, A.: A paksi pleistocén üledékek esigái és értékelesük (Snails of the Pleistocene sedimentations in Paks and their valuation). - Allattani Közlemények, 3-4, 171-188, Budapest 1954.

Kriván, P.: Die klimatische Gliederung des mitteleuropäischen Pleistozäns. - Acta geologica, 3, H. 4, 357-382, Budapest 1955 (1955a).

- : La division climatologique du Pleistocene en Europe centrale et le profil de loess de Paks. A Magyar Allami Földtáni Intézet Evkönyve, 43, 3, 441-512, Budapest 1955 (1955b).

Kubiena, W. L.: Zur Mikromorphologie, Systematik und Entwicklung der rezenten und fossilen Lößböden. - Eiszeitalter u. Gegenwart, 7, 102-112, Öhringen 1956.

- : Zur Mikromorphologie und Mikromorphogenese der Lößböden Neuseelands. In: Soil Micromorphology (Ed. A. Jongerius), 219-235, Amsterdam 1964.

LožEK, V.: Das Problem der Lößbildung und die Lößmollusken. Eiszeitalter u. Gegenwart, 16, 61-75, OOhringen 1965.

PÉCSI, M.: Zur Frage der Typen der Lösse und lößartigen Sedimente im Karpatenbecken und ihrer lithostratigraphischen Einteilung. - Földrajzi Közlemények, 89, 305-323, Budapest 1965 (1965a).

- : Genetic Classification of the Deposits constituting the Loess Profiles of Hungary. - Acta Geologica Hung., 9, 65-84, Budapest 1965 (1965b).

- : Der Lößaufschluß von Mende. - Földrajzi Közlemények, 89, 332-338, Budapest 1965 (1965c).

- : Lösse und lößartige Sedimente im Karpatenbecken und ihre lithostratigraphische Gliederung. - Peterm. Geogr. Mitt. 110, 176-189 und 241-252, Gotha 1966.

- : Horizontal and vertical Distribution of the Loess in Hungary. - Studia Geomorphologica Carpatho - Balcanica, 1, 13-20, Krakow 1967.

Rohdenburg, H.: Ein Beitrag zur Deutung des „Gefleckten Horizontes“. - Eiszeitalter u. Gegenwart, 15, 66-71, Ohringen 1964.

Rohdenburg, H. \& Meyer, B.: Zur Feinstratigraphie und Paläopedologie des Jungpleistozäns nach Untersuchungen an südniedersächsischen und nordhessischen Lößprofilen. - Mitt. Dtsch. Bodenkundl. Ges., 5, 1-137, Göttingen 1966.

Scheffer, F., Meyer, B. \& Gebhardt, H.: Pedochemische und kryoklastische Verlehmung (Tonbildung) in Böden aus kalkreichen Lockersedimenten (Beispiel Löß). — Z. Pflanzenern. Düng., Bodenkunde, 114, 77-89, Weinheim 1966.

SCHERF, E.: Versuch einer Einteilung des ungarischen Pleistozäns auf moderner, polyglazialistischer Grundlage. - Verhandl. der II. Internat. Quartärkonferenz 1936, Wien 1938.

Schlichting, E. \& Blume, H. P.: Art und Ausmaß der Veränderungen des Bestandes mobiler Oxide in Böden aus jungpleistozänem Geschiebemergel und ihren Horizonten. - Z. Pflanzenernähr., Düng., Bodenkunde, 96, 144-156, Weinheim 1962.

Short Guide to Soil-Excursion from Moscow to Kherson. - ("Kolos" Publishing House) 94 S., Moskau 1964.

SCHROEDER, D.: Untersuchungen über Verwitterung und Bodenbildung an Lößprofilen. - Habil.Schrift, 84 S., Hannover 1954.

- : Mineralogische Untersuchungen an Lößprofilen. - Heidelberger Beiträge z. Mineralogie und Petrographie, 4, 443-463, Heidelberg 1955.

Schwertmann, U.: Differenzierung der Eisenoxide des Bodens durch photochemische Extraktion mit saurer Ammoniumoxalat-Lösung. - Z. Pflanzenernähr., Düng., Bodenkunde, 105, 194-202, Weinheim 1964.

Sмоцíкоví, L.: Mikromorphologie der altpleistozänen Fossilböden von Cservený kopec bei Brno (Brünn). - Vestnik Ustredniho ústavo geologického, 42, 369-373, Prag 1967.

- : Mikromorphologie und Mikromorphometrie der pleistozänen Bodenkomplexe (Vergleichsuntersuchungen der Interglazialböden von Letky nad Vltavou). - Rozpravy Ceskoslovenské Akademie Ved. Rada Matematických a Privod nich Ved, Rocnik, 78, 2, 47 S., Prag 1968.

Stefanovits, P.: Az agyagos rész viszgálata jelemzö talajtipusokhan (Investigation of the clay fraction of characteristical soil types). - Agrokémia es Talajtan, 8, 37 ff., Budapest 1959 (1959a).

- : A magyarországi erdötalajok genetikus - taljaföldrajzi osztályozaga (Genetisch-bodengeographische Klassifikation der Waldböden Ungarns). - Agrokémia és Talajtan, 8, 163 184, Budapest 1959 (1959b).

- : Untersuchungsangaben der begrabenen Bodenschichten im Lößprofil von Mende. - Földrajzi Közlemények, 89, 339-344, Budapest 1965.

Stefanovits, P. \& Szücs, L.: Magyarország Genetikus Talajtérképe (Genetische Bodenkarte Ungarns). Mit Erl. v. P. Stefanovits, Budapest 1961. 
Stefanovits, P. \& RoszavöLgyi, J.: Ujabb paleopedológiai adatok a paksi szelvényrôl. (Weitere paläopedologische Angaben über das Bodenprofil von Paks). - Agrokémia és talajtan, 11, 143-160, Budapest 1962.

— : Beschreibung des Lößprofils bei der Ziegelei Paks. - Földrajzi Közlemények, 89, 357-360, Budapest 1965.

Szücs, L.: A hazai Csernozjom talajok osztályozása (Klassifikation der Tschernosemböden Ungarns). - Agrokémia és Talajtan, 8, 83 ff., Budapest 1959.

Zagwijn, W. \& Paepe, R.: Die Stratigraphie der weichselzeitlichen Ablagerungen der Niederlande und Belgiens. - Eiszeitalter u. Gegenwart, 19, 129-146, Budapest 1968.

Manuskr. eingeg. 19. 12. 1969.

Anschrift des Verf.: Dr. A. Bronger, Geographisches Institut der Universität Kiel, 23 Kiel, Olshausenstraße 40-60. 